\title{
Ecological status and traditional knowledge of medicinal plants in Kedarnath Wildlife Sanctuary of Garhwal Himalaya, India
}

\author{
Jahangeer A Bhat ${ }^{1}$, Munesh Kumar ${ }^{1 *}$ and Rainer W Bussmann ${ }^{2}$
}

\begin{abstract}
Background: Himalayan forests are the most important source of medicinal plants and with useful species for the local people. Kedarnath Wildlife Sanctuary (KWLS) is situated in the interior part of the Garhwal Himalayan region. The presented study was carried out in Madhmeshwar area of KWLS for the ecological status of medicinal plants and further focused on the ethnomedicinal uses of these plants in the study area.

Methods: Ecological information about ethnomedicinal plants were collected using random quadrats in a random sampling technique along an altitudinal gradient in the KWLS. Information on medicinal properties of plants encountered in the present study was generated by questionnaire survey and was also compared with relevant literature.

Results: A total of 152 medicinally important plant species were reported, in which 103 were found herbs, 32 shrubs and 17 were tree species which represented 123 genera of 61 families. A total of 18 plant species fell into the rare, endangered (critically endangered) and vulnerable status categories.

Conclusion: The present study documented the traditional uses of medicinal plants, their ecological status and importance of these plants in the largest protected area of Garhwal Himalaya. This study can serve as baseline information on medicinal plants and could be helpful to further strengthen the conservation of this important resource.
\end{abstract}

Keywords: Ethnomedicinal plants use, Ecological status, Resources, Altitudinal zone

\section{Introduction}

The forests of India have been the source of traditional medicines for millennia. Of the 17,000 species of higher plants described in India, 7500 are known for their medicinal uses [1]. The Charak Samhita, a document on herbal therapy written about 300 BC, reports on the production of 340 herbal drugs and their indigenous uses [2]. The use of alternative medicine is growing because of its moderate costs and increasing faith in herbal medicine. Allopathic medicine can cure a wide range of diseases, however, its high prices and side-effects are causing many people to return to herbal medicines which tend to have fewer side effects [3]. A great amount of traditional

\footnotetext{
* Correspondence: muneshmzu@yahoo.com

${ }^{1}$ Department of Forestry and Natural Resources, H.N.B, Garhwal University, Srinagar-Garhwal, Uttarakhand 249161, India

Full list of author information is available at the end of the article
}

knowledge about the use of medicinal plant species is still carried and orally transmitted by indigenous peoples. Regions with less accessibility and a comparatively slow rate of development, such as and mountainous areas like the Himalayas are excellent examples [4,5]. Because of the fast acceleration of market demand for herbal medicines, and recent controversies related to access, benefit sharing and biopiracy, the documentation of indigenous knowledge is of urgent priority [6-10]. Indigenous knowledge, supplemented by the latest scientific insights, can offer new holistic models of sustainable development that are economically viable, environmentally benign and socially acceptable [11]. Currently, approximately $25 \%$ of allopathic drugs are derived from plant based compounds, and many others are synthetic analogues built on prototype compounds isolated from plant species [12]. According to the World Health Organization (WHO), as many as 
$80 \%$ of the world's people depend on traditional medicine to meet their primary health care needs [13].

The Himalayan range in the northern part of India harbours a great diversity of medicinal plants. Of the approximately 8000 species of angiosperms, 44 species of gymnosperms and 600 species of pteridophytes that have been reported in the Indian Himalaya [14], 1748 species are known for their medicinal properties [15]. The state of Uttarakhand is a part of north-western Himalaya, and still maintains a dense vegetation cover (65\%). The maximum species of medicinal plants have been reported from Uttarakhand [16,17], followed by Sikkim and North Bengal [15]. The trans-Himalaya in contrast sustains about 337 species of medicinal plants [4], which are low compared to other areas of the Himalaya due to the distinct geography and ecological marginal conditions [18]. Recent years have seen a sudden rise in the demand of herbal products and plant based drugs across the world resulting in the heavy exploitation of medicinal plants. Habitat degradation, unsustainable harvesting and over-exploitation to meet the demands of the mostly illegal trade in medicinal plants have already led to the extinction of more than 150 plant species in the wild [19]. More than $90 \%$ of plant species used in the herbal industries are extracted from the wild, and about $70 \%$ of the medicinal plants of Indian Himalaya are subject to destructive harvesting [20,21], and the majority of these plants stems from sub-alpine and alpine regions of the Himalaya [21]. The importance of ethnobiological knowledge on species-ecology can provide leads for new paths in scientific research and conservation, and has received growing attention in resource management worldwide [22,23]. International agencies such as the World Wildlife Fund (WWF) and United Nations Educational, Scientific and Cultural Orga-nization (UNESCO) as part of their people and plants initiative, are promoting research on ethnobotanical knowledge and the integration of people's perceptions and practices in resource management at the local level [24].

The Kedarnath Wildlife Sanctuary (KWLS) is rich in biological diversity and is one of the most important regions of Garhwal Himalaya. The area of KWLS selected for this study is a particularly remote area, and the villagers residing in the area are fully dependent on forest resources, especially ethnomedicines for their daily livelihoods. Some ethnomedicinal studies on plants in this part of Himalayan region have been published, but hardly any ecological studies have also been carried out. The aim of the present study was to assess the ecological status of ethnomedicinal plants in a part of the largest protected area of Garhwal Himalaya.

\section{Materials and methods}

\section{Study area}

The present study was carried out in Madhmeshwar area, which is the interior part of Kedarnath Wildlife Sanctuary (KWLS) in the Western Himalaya of Chamoli-Rudraprayag districts of Uttarakhand, India. KWLS was established in 1972 and is situated in the north-eastern part of the Garhwal Himalayas between $30^{\circ} 25^{\prime}-30^{\circ} 41^{\prime} \mathrm{N}, 78^{\circ} 55^{\prime}$ $79^{\circ} 22^{\prime}$ E. The Sanctuary falls under the IUCN management Category IV (Managed Nature Reserve) in the Biogeographical Province 2.38.12 of Himalayan highlands. KWLS is one of the largest protected areas with 97517.80 ha (25293.70 ha in Chamoli district and 72224.10 ha in Rudraprayag district) in the Western Himalaya [19]. The sanctuary lies in the upper catchment of the Alaknanda and Mandakini Rivers, which are major tributaries of Ganges. It is bordered by high mountain peaks, e.g. Kedarnath $(6940 \mathrm{~m})$, Mandani $(6193 \mathrm{~m})$ and Chaukhamba $(7068 \mathrm{~m})$ and harbors extensive alpine meadows, in particular Trijuginarayan, Kham, Mandani, Pandavshera, Manpai and Bansinarayan in the north, and several dense broad leaved oak mixed forest stands in the south. The present study represents data from a transect from the base of the mountain to the top in the Madhmaheshwer area between the coordinates $30^{\circ} 35^{\prime} 42^{\prime}-30^{\circ} 38^{\prime} 12^{\prime} \mathrm{N}, 79^{\circ} 10^{\prime} 00^{\prime}-79^{\circ} 13^{\prime} 00^{\prime} \mathrm{E}$ (Figure 1). The area receives $3000 \mathrm{~mm}$ of annual precipitation, of about $60 \%$ fall during the monsoon season (June-August). The relative humidity varies from 35 to $85 \%$ annually. There is moderate to heavy snowfall during DecemberFebruary, even in low-altitude areas. The mean maximum temperature varies between $4^{\circ} \mathrm{C}$ (January) and $33.5^{\circ} \mathrm{C}$ (June).

\section{Ecological analysis of plant species}

The vegetation analysis of ethnomedicinal plants was carried out following the stratified random sampling technique involving random quadrats. The size of the square plots was $100 \mathrm{~m}^{2}$ for trees, and nested within the main quadrats two plots of $25 \mathrm{~m}^{2}$ for shrubs and four plots of $1 \mathrm{~m}^{2}$ for herbs. The study area was divided into five altitudinal zones along the altitudinal gradient, to assess the ecological status of medicinal plant species. The frequency and density of all species was determined $[25,26]$.

\section{Ethnomedicinal study of plant species}

The plant species reported in the ecological studies of Madhmeshwar area were only taken for the ethnomedicinal study. A well structured questionnaire was prepared covering different questions regarding plants used for ethnomedicinal purposes. For the ethnomedicinal study only two villages were observed i.e., Gundhaar and Ransi in Madhmeshwar area and the respondents were selected 


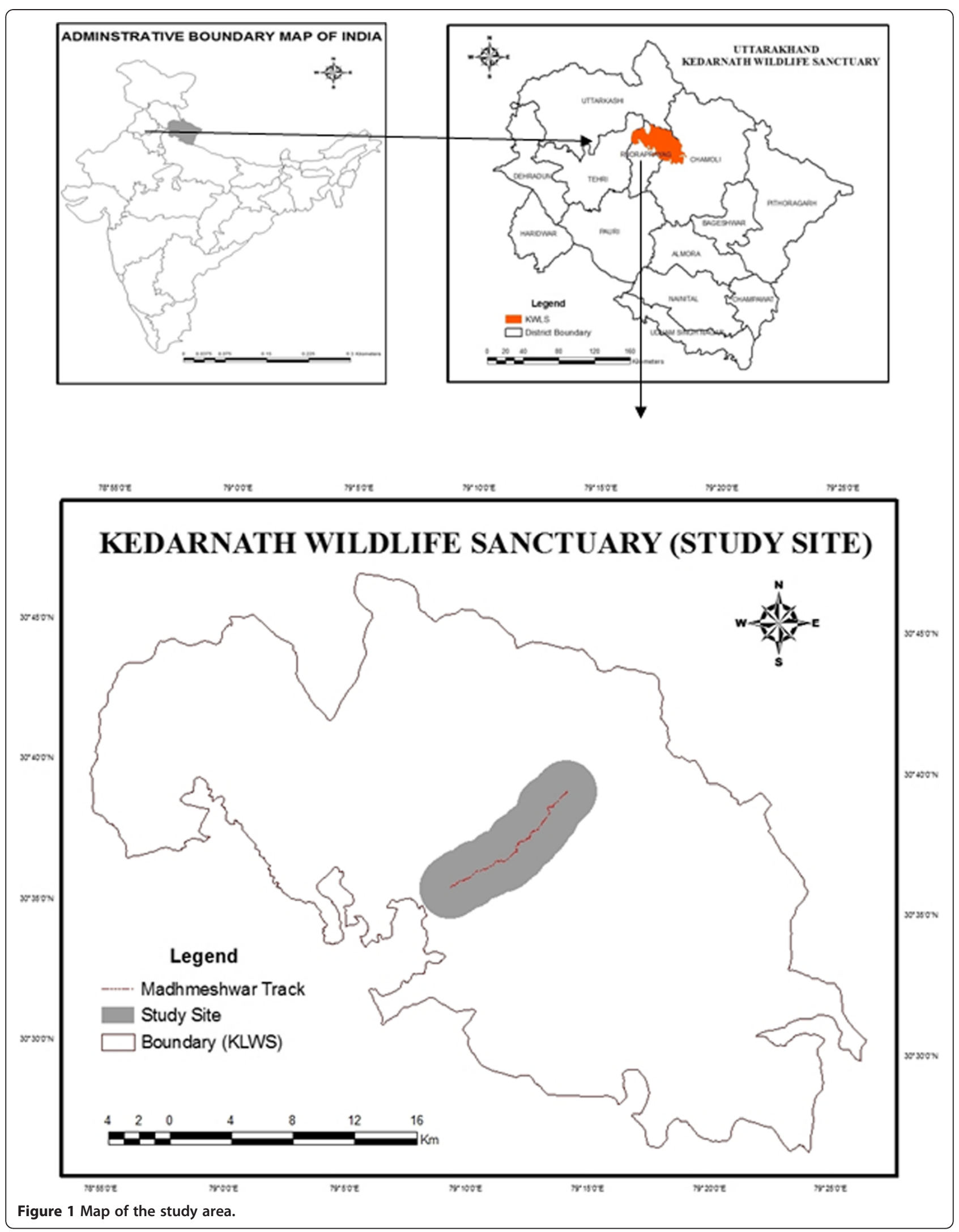


Table 1 Plant species with their status and the part used in different ailments

\begin{tabular}{|c|c|c|c|c|c|}
\hline Scientific name & Accession No. & Status & Habit & Plant part used & Medicinal uses \\
\hline Abies pindrow Royle. & JAB-GUH-20578 & & $T$ & Bark extract $^{2}$ & 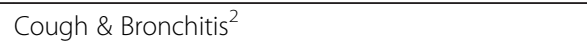 \\
\hline Aconitium hetrophyllum Wallich & JAB-BSD-114039 & $\mathrm{R}^{1}, \mathrm{Ce}^{3}, V \mathrm{u}^{6}$ & $\mathrm{H}$ & $\operatorname{Root}^{5}$ & Fever, cough,stomachache ${ }^{5}$ \\
\hline Aesculus indica (Wall. ex Cambess.) Hook.f. & JAB-GUH-20435 & & $\mathrm{T}$ & Seed paste ${ }^{2}$ & Rheumatic Pain ${ }^{2}$ \\
\hline Ainsliaea apetra DC. & JAB-GUH-20677 & & $\mathrm{H}$ & Root extract $^{2}$ & Fever, painful urination ${ }^{2}$ \\
\hline Ainsliaea latifolia (D.Don) Sch.-Bip. & JAB-GUH-20680 & & $\mathrm{H}$ & Root decoction ${ }^{2}$ & Colic $^{2}$ \\
\hline Anaphalis contorta (D.Don) Hook.f. & JAB-GUH-20437 & & $\mathrm{H}$ & 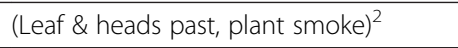 & (Cuts, wounds \& boils, insect repellent) $^{2}$ \\
\hline Anaphalis margaritaceae (L.) Benth & JAB-GUH-20458 & & $\mathrm{H}$ & 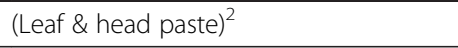 & (Cuts, wounds \& boils) $^{2}$ \\
\hline Anaphalis triplinervis (Sims.) C.B. Clarke & JAB-GUH-20453 & & $\mathrm{H}$ & Leaf juice $^{2}$, Flower ${ }^{5}$ & Laceration of toes ${ }^{2}$, Dressing wounds ${ }^{5}$ \\
\hline Anemone obtusiloba D.Don & JAB-GUH-20619 & & $\mathrm{H}$ & Root decoction ${ }^{2}$ & Diarrhoea $^{2}$ \\
\hline \multirow[t]{2}{*}{ Anemone rivularis Buch.-Ham. ex DC } & JAB-GUH-20613 & & $\mathrm{H}$ & ${\text { (Leaf past \& juice) })^{2} \text { Leaves }^{5}}$ & (Wounds $^{5}$, sores \& ear ache in local therapy) ${ }^{2}$ \\
\hline & JAB-BSD-114043 & & & & \\
\hline Arachne cordifolia (Decne.) Hurusawa & JAB-GUH-20527 & & S & Leaf \& Stem paste ${ }^{2}$ & Wounds \& Antidote to snake bite ${ }^{2}$ \\
\hline Arisaema jacquemontii Blume & JAB-GUH-20432 & & $\mathrm{H}$ & Fruits $^{2}$, Tuber $^{5}$ & $\begin{array}{l}\text { (Antidote of poisonous mushrooms \& snake bite) }{ }^{2} \text {, } \\
\text { (Cough, kidney \&skin diseases) }^{5}\end{array}$ \\
\hline Artemisia japonica Thunb. & JAB-GUH-20446 & & $\mathrm{H}$ & (Leaves \& flower tops) $^{2}$ & Incense \& insecticide ${ }^{2}$ \\
\hline Artemisia roxburghiana Bess. & JAB-GUH-20468 & & $\mathrm{H}$ & Plant extract ${ }^{2}$ & (Antipyretic, Tonic \& also rubbed on skin allergy) ${ }^{2}$ \\
\hline \multirow[t]{2}{*}{ Asparagus filicinus Buch.-Ham. ex D. Don } & JAB-GUH-20436 & & $\mathrm{H}$ & Root tuberous ${ }^{2}$ & (Diabetes, diarrhoea \& dysentery) $)^{2}$ \\
\hline & JAB-BSD-114062 & & & & \\
\hline Aster peduncularis Wallich & JAB-GUH-20687 & & $\mathrm{H}$ & (Plant extract \& Root powder) $^{2}$ & 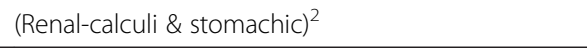 \\
\hline Barleria cristata $\mathrm{L}$. & JAB-GUH-20417 & & $\mathrm{H}$ & $\left(\right.$ Root decoction, Root \& Leaves paste) ${ }^{2}$ & 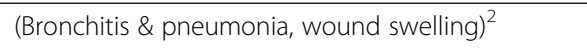 \\
\hline Begonia picta Smith & JAB-GUH-20411 & & $\mathrm{H}$ & Plant decoction ${ }^{2}$ & Colic \& dyspepsia ${ }^{2}$ \\
\hline Bergenia ciliata (Haw.) Sternb. & JAB-GUH-20650 & & $\mathrm{H}$ & (Root $^{5}$ rhizomatous $)^{2}$ & $\begin{array}{l}\text { (Tonic, febrifuge, digestive \& cutaneous disorders) }{ }^{2}, \\
\text { (Fevers, diarrhoea \& pulmonary infections) })^{5}\end{array}$ \\
\hline Bidens bipinnata $\mathrm{L}$. & JAB-GUH-20440 & & $\mathrm{H}$ & Leaf juice $^{2}$ & 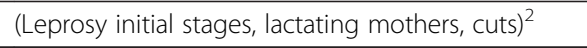 \\
\hline Bidens biternata (Lour.) Merr. \& Sherff & JAB-GUH-20441 & & $\mathrm{H}$ & Leaf juice $^{2}$ & ${\text { (Leprosy initial stages, lactating mothers, cuts) }{ }^{2}}^{2}$ \\
\hline Bidens pilosa $\mathrm{L}$. & JAB-GUH-20444 & & $\mathrm{H}$ & (Plant extract \& herbs of plants) $^{2}$ & $\left(^{(C o u g h} \& \text { Bronchitis, leucoderma }\right)^{2}$ \\
\hline Bistorta amplexicaulis (D.Don) Greene & JAB-GUH-20600 & & $\mathrm{H}$ & ${\text { (Plant decoction \& Leaf paste) })^{2}}^{2}$ & (Cause abortion, wounds \& relieves dysentery) ${ }^{2}$ \\
\hline Bistorta vaccinifolia (Wall. ex Meisn.) Greene. & JAB-BSD-114056 & $R^{2}$ & $\mathrm{H}$ & Root decoction ${ }^{2}$ & Tuberculosis $^{2}$ \\
\hline Blumea lanceolaria (Roxb.) Druce & JAB-GUH-20679 & & $\mathrm{H}$ & Leaf paste $^{2}$ & Wounds \& cuts ${ }^{2}$ \\
\hline Buddleja asiatica Lour. & JAB-GUH-20485 & & S & Leaf extract \& Roots $^{2}$ & Skin diseases \& Abortifacient ${ }^{2}$ \\
\hline Bupleurum falcatum L. & JAB-GUH-20427 & & $\mathrm{H}$ & Root decoction ${ }^{2}$ & Fever \& liver troubles $^{2}$ \\
\hline Calanthe tricarinata Lindl. & JAB-GUH-20573 & & $\mathrm{H}$ & (Leaf paste Leaves \& Pseudo-bulbs) $^{2}$ & ${\text { (Sores \& eczema, aphrodisiac) })^{2}}$ \\
\hline Callicarpa arborea Roxb. & JAB-GUH-20672 & & $T$ & Bark $^{2}$ & Skin ailments ${ }^{2}$ \\
\hline Cannabis sativa $\mathrm{L}$. & JAB-GUH-20488 & & $\mathrm{H}$ & Flowers $^{2}$ & Intoxicating agent ${ }^{2}$ \\
\hline Carpinus viminea Lindl. & JAB-GUH-20503 & & $T$ & Leaves $^{5}$ & Bone fracture ${ }^{5}$ \\
\hline
\end{tabular}


Table 1 Plant species with their status and the part used in different ailments (Continued)

\begin{tabular}{|c|c|c|c|c|c|}
\hline Clematis buchananiana DC. & JAB-GUH-20611 & & $\mathrm{S}$ & Leaf paste $^{2}$ & Skin ailments ${ }^{2}$ \\
\hline Clematis montana Buch.-Ham. ex DC. & JAB-GUH-20618 & & $\mathrm{H}$ & Leaf extract $^{2}$ & Diabetes \& urinary troubles ${ }^{2}$ \\
\hline Clinopodium umbrosum (M.Bieb.) C. Koch & JAB-GUH-20558 & & $\mathrm{H}$ & ${\text { (Plant extract \& Leaf infusion) })^{2}}$ & $\begin{array}{l}\text { (Astringent, carminative, Blood purifier \& Gastric } \\
\text { troubles) }^{2}\end{array}$ \\
\hline Corallodiscus lanuginosus (Wall. ex DC.) B.L. Burtt & JAB-BSD-114064 & & $\mathrm{H}$ & Leaves $^{2}$ & Kidney stone ${ }^{2}$ \\
\hline Coriaria nepalensis Wallich & JAB-GUH-20502 & $R^{2}$ & S & Fruits $^{2}$ & Emetic $^{2}$ \\
\hline Cotoneaster microphyllus Wall. ex Lindl. & JAB-GUH-20640 & & $\mathrm{S}$ & Leaf, Fruits \& Root Paste ${ }^{2}$ & Diarrhoea, Cuts \& Wounds ${ }^{2}$ \\
\hline Cyathula capitata Moq. & JAB-GUH-20422 & & $\mathrm{H}$ & $(\text { Leaf extract \& Urticle })^{2}$ & Emetic \& abortifacient $^{2}$ \\
\hline Cyathula tomentosa Moq. & JAB-GUH-20421 & & $\mathrm{H}$ & Leaf extract $^{2}$ & 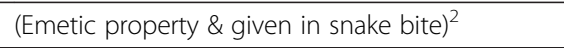 \\
\hline Cynoglossum glochidiatum Wall. ex Benth. & JAB-BSD-114059 & & $\mathrm{H}$ & Root extract $^{2}$ & Dyspepsia \& digestive disorders $^{2}$ \\
\hline Cynoglossum lanceolatum Forssk. & JAB-GUH-20481 & & $\mathrm{H}$ & Plant infusion ${ }^{2}$ & Cold \& cough ${ }^{2}$ \\
\hline Debregeasia salicifolia (D.Don) Rendle & JAB-GUH-20666 & & $\mathrm{S}$ & Bark $^{2}$ & Plaster for Bone Fracture ${ }^{2}$ \\
\hline Delphinium vestitum Wall. ex Royle & JAB-GUH-20616 & & $\mathrm{H}$ & Plant Stem ${ }^{5}$ & Body swelling ${ }^{5}$ \\
\hline Desmodium elagans DC. & JAB-GUH-20531 & & $\mathrm{S}$ & Root infusion \& Roots ${ }^{2}$ & Epilepsy \& Carminatives ${ }^{2}$ \\
\hline Deutzia compacta Craib. & JAB-GUH-20541 & & $\mathrm{S}$ & Leaves $^{2}$ & Diuretic $^{2}$ \\
\hline Dicliptera bupleuroides Nees & JAB-GUH-20418 & & $\mathrm{H}$ & (Leaf $^{5}$ paste \& juice $)^{2}$ & $\begin{array}{l}\text { (Wounds cough \& gastro-enteritis) })^{2} \text { (Fever, skin } \\
\text { diseases \& stomachache) }\end{array}$ \\
\hline Dipsacus inermis Wallich & JAB-GUH-20483 & & $\mathrm{H}$ & Root paste $^{2}$ & Leucoderma \& contusions $^{2}$ \\
\hline Elephantopus scaber $\mathrm{L}$. & JAB-GUH-20448 & & $\mathrm{H}$ & $(\text { Root extract \& leaves) })^{2}$ & (Fever, stops vomiting, tonic for blood diseases) $)^{2}$ \\
\hline Elsholtzia fruticosa (D.Don) Rehder. & JAB-GUH-20551 & & $\mathrm{S}$ & Seeds $^{2}$ & Sciatica reliever $^{2}$ \\
\hline Elsholtzia strobilifera Benth. & JAB-GUH-20549 & & $\mathrm{H}$ & (Plant $^{5}$ paste $)^{2}$ & (Bruises \& wounds $\left.^{5}\right)^{2}$ \\
\hline Eupatorium odenophorum Spreng. & JAB-GUH-20452 & & $\mathrm{s}$ & Leaves $^{2,5}$ & Wounds $^{2}$, Skin diseases ${ }^{5}$ \\
\hline Euphorbia chamaesyce L. & JAB-GUH-20410 & & $\mathrm{H}$ & Plant juice $^{2}$ & ${\text { (Constipation \& dysentery to infants) })^{2}}^{2}$ \\
\hline Euphorbia hypericifolia L. & JAB-GUH-20529 & & $\mathrm{H}$ & Leaf infusion $^{2}$ & ${\text { (Dysentery, diarrhoea, menorrhagia) }{ }^{2}}^{2}$ \\
\hline Euphorbia pilosa Linn. & JAB-GUH-20528 & & $\mathrm{H}$ & (Root decoction \& Fruits) $^{2}$, Seed \& Leaves ${ }^{5}$ & Constipation \& emetic $^{2}$, Food poisoning ${ }^{5}$ \\
\hline Fagopyrum dibotrys (D.Don) Hara & JAB-GUH-20597 & & $\mathrm{H}$ & Leaf paste $^{2}$ & Insect bite ${ }^{2}$ \\
\hline Fragaria nubicola Lindl. ex Lacaita & JAB-GUH-20628 & & $\mathrm{H}$ & Leaf juice $^{2}$ & Ear ache $^{2}$ \\
\hline Galinsoga parviflora Cav. & JAB-GUH-20697 & & $\mathrm{H}$ & Plant extract ${ }^{2}$ & Antidote of nettle sitting $^{2}$ \\
\hline Galium aparine L. & JAB-GUH-20646 & & $\mathrm{H}$ & 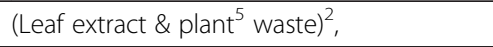 & Astringent $^{5}$, skin diseases ${ }^{2}$ \\
\hline Galium asperifolium Wallich. & JAB-GUH-20648 & & $\mathrm{H}$ & Plant waste ${ }^{2}$ & Skin ailments ${ }^{2}$ \\
\hline Geranium wallichianum D. Don ex Sweet & JAB-BSD-114067 & & $\mathrm{H}$ & Root $^{5}$ juice $^{2}$ & 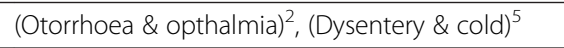 \\
\hline \multirow[t]{2}{*}{ Gerbera gossypina (Royle) P. Beauv. } & JAB-GUH-20449 & & $\mathrm{H}$ & ${\text { (Leaf juice \& paste) })^{2}}$ & ${\text { (Cuts, wounds, plaster on bone fracture) }{ }^{2}}^{2}$ \\
\hline & JAB-BSD-114060 & & & & \\
\hline Girardiana diversifolia (Link) Friis & JAB-GUH-20670 & & $\mathrm{H}$ & Leaf juice ${ }^{2}$, Plant whole ${ }^{5}$ & Gonorrhoea $^{2}$, Diuretic ${ }^{5}$ \\
\hline Gonatanthus pumilus (D.Don) Engl. \& Krause & JAB-GUH-20431 & & $\mathrm{H}$ & Root tuber paste ${ }^{2}$ & Burns \& wounds ${ }^{2}$ \\
\hline Gonostegia hirta (Blume) Miq & JAB-GUH-20669 & & $\mathrm{H}$ & Roots $^{2}$ & Plaster on fractured bones ${ }^{2}$ \\
\hline
\end{tabular}


Table 1 Plant species with their status and the part used in different ailments (Continued)

\begin{tabular}{|c|c|c|c|c|c|}
\hline Hippophae salicifolia D.Don & JAB-GUH-20520 & & $\mathrm{T}$ & Fruits $^{2,5}$ & ${\text { (Dandruff })^{2} \&(\text { Cardiac trouble })^{5}}^{5}$ \\
\hline Holmskioldia sanguinea Retz. & JAB-GUH-20673 & & $S$ & Leaf paste $\&$ Roots $^{2}$ & Body Swelling \& Febrifuge ${ }^{2}$ \\
\hline Hypericum choisianum Wall. ex N. Robson & JAB-GUH-20691 & $R^{2}$ & $S$ & Leaf powder ${ }^{2}$ & Fever $^{2}$ \\
\hline Impatiens scabrida DC. & JAB-GUH-20474 & & $\mathrm{H}$ & Plant Stem ${ }^{2}$ & Cause abortion $^{2}$ \\
\hline Indigofera heterantha Wall. ex Brandis & JAB-GUH-20532 & & $S$ & Leaf juice $^{2}$ & Diarrhoea, Dysentery \& Cough ${ }^{2}$ \\
\hline Inula cappa (Buch.-Ham. ex D. Don) DC. & JAB-GUH-20456 & & $S$ & Roots $^{2}$ & Suppressed urination $^{2}$ \\
\hline Juglans regia $\mathrm{L}$. & JAB- GUH-20520 & & $\mathrm{T}$ & Leaves $^{2},(\text { Bark \& Roots })^{5}$ & Fungicide \& Insecticide $^{2}$, Tooth ache ${ }^{5}$ \\
\hline Jurinea dolomiaea Boiss. & JAB-GUH-20443 & $E^{3}$ & $\mathrm{H}$ & Root $^{5}$ & 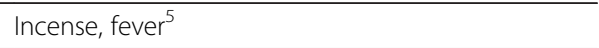 \\
\hline Lamium album $\mathrm{L}$. & JAB-GUH-20559 & & $\mathrm{H}$ & Plant decoction ${ }^{2}$, Flower ${ }^{5}$ & Contraceptive $^{2}$, Bleeding after childbirth ${ }^{5}$ \\
\hline Leptodermis Lanceolata Wallich & JAB-GUH-20643 & & S & Bark paste $^{2}$ & Migraines $^{2}$ \\
\hline Leucas lanata Benth. & JAB-GUH-20553 & & $\mathrm{H}$ & Plant infusion ${ }^{2}$ & Whooping cough ${ }^{2}$ \\
\hline Leycesteria formosa Wallich & JAB-GUH-20494 & & $\mathrm{S}$ & Leaf paste $^{2}$ & Dandruff \& Lice in hair $^{2}$ \\
\hline Lindenbergia indica (L.) Vatke & JAB-GUH-20656 & & $\mathrm{H}$ & Leaves $^{2}$ & (Bronchitis, Cuts \& wounds) $^{2}$ \\
\hline Lonicera angustifolia Wall. ex DC. & JAB-GUH-20495 & & $\mathrm{S}$ & Fruits $^{2}$ & Gastric troubles of cattle ${ }^{2}$ \\
\hline Lyonia ovalifolia (Wallich) Drude & JAB-GUH-20524 & & $\mathrm{T}$ & Seed paste ${ }^{2}$ & Wounds \& Boils ${ }^{2}$ \\
\hline Maianthemum purpureum (Wall.) La Frankie & JAB-GUH-20565 & & $\mathrm{H}$ & Leaf extract $^{2}$ & Dysmenorrhoea $^{2}$ \\
\hline Morina longifolia Wall. ex DC. & JAB-GUH-20571 & $R^{2}$ & $\mathrm{H}$ & $\left(\text { Root }^{5} \text { paste \& dried roots) }\right)^{2}$ & 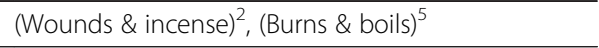 \\
\hline Myrica esculenta Buch.-Ham. ex D. Don & JAB-GUH-20702 & & $T$ & Bark $^{2,4}$, Leaves ${ }^{5}$ \& Fruit 4,5 & $\begin{array}{l}\text { (Intoxicate to fishes) })^{2} \text { (Vit. C, Asthama, Bronchitis, } \\
\text { Diarrhoea \& tooth ache) })^{4}(\text { Skin diseases \& wounds })^{5}\end{array}$ \\
\hline Neolitsea pallens (D.Don) Momiyama \& Hara & JAB-GUH-20563 & & $\mathrm{T}$ & Fruits $^{2}$ & Scabies \& Eczema ${ }^{2}$ \\
\hline Nepeta ciliaris Benth. & JAB-GUH-20552 & & $\mathrm{H}$ & 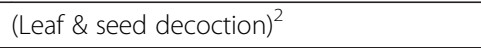 & Fever $^{2}$ \\
\hline Nomocharis oxypetala (Royle.) E.H.Wilson. & JAB-GUH-20557 & & $\mathrm{H}$ & Bulb $^{5}$ & Vigorous $^{5}$ \\
\hline Origanum vulgare $\mathrm{L}$. & JAB-GUH-20561 & & $\mathrm{H}$ & Plant extract ${ }^{2}$, Leaves ${ }^{5}$ & 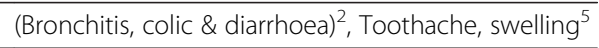 \\
\hline Paeonia emodii Wall. ex Royle & JAB-GUH-20575 & & $\mathrm{H}$ & 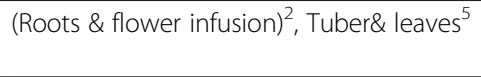 & $\begin{array}{l}\text { (Whooping cough, diarrhoea, intestinal spasms) }{ }^{2} \text {, } \\
\text { Uterine diseases }\end{array}$ \\
\hline Parnassia nubicola Wall. ex Royle & JAB-GUH-20539 & & $\mathrm{H}$ & Root $^{5}$ paste $^{2}$ & Antidote of snake bite ${ }^{2}$, Boils ${ }^{5}$ \\
\hline Pedicularis hoffmeisteri Klotz. & JAB-GUH-20657 & & $\mathrm{H}$ & Plant whole $^{5}$ & Food poisoning $^{5}$ \\
\hline Persicaria polystachya (Wall. ex Meissn.) H. Gross & JAB-GUH-20598 & & $\mathrm{S}$ & Leaf paste $^{2}$ & Laceration of toes ${ }^{2}$ \\
\hline Phalaris minor Retz. & JAB-GUH-20591 & & $\mathrm{H}$ & Root paste ${ }^{2}$ & Wounds $^{2}$ \\
\hline Picrorhiza kurrooa Royle ex Benth. & JAB-GUH-20654 & $R^{1}, C E^{3}, V u^{6}$ & $\mathrm{H}$ & Root $^{5}$ & Fever, stomachache ${ }^{5}$ \\
\hline Pimpinella acuminata (Edgew.) C.B. Clarke & JAB-GUH-20428 & & $\mathrm{H}$ & Plant extract ${ }^{2}$ & Diarrhoea \& dysentery ${ }^{2}$ \\
\hline Pimpinella diversifolia DC. & JAB-GUH-20426 & & $\mathrm{H}$ & Plant extract ${ }^{2}$ & (Digestive disorders, cold \& cough) $^{2}$ \\
\hline Pinus roxburghii Sargent & JAB-GUH-20701 & & $\mathrm{T}$ & Saw Dust ${ }^{2} \&$ Aerial parts ${ }^{4}$ & (Asthma \& Bronchitis) $^{2}$, (Resin for cracked toes) $)^{4}$ \\
\hline Plantago depressa Willd. & JAB-GUH-20580 & & $\mathrm{H}$ & $(\text { Leaf \& seed paste) })^{2}$ & (Cuts, wounds, piles) $^{2}$ \\
\hline Plantago himalaica Pilger. & JAB-GUH-20579 & & $\mathrm{H}$ & Leaves $^{5}$ & Dysentery $^{5}$ \\
\hline Podophyllum hexandrum Royle. & JAB-GUH-20592 & $R^{1}, E^{3,} E^{6}$ & $\mathrm{H}$ & Root $^{5}$ & Wounds ${ }^{5}$ \\
\hline
\end{tabular}


Table 1 Plant species with their status and the part used in different ailments (Continued)

\begin{tabular}{|c|c|c|c|c|c|}
\hline Polygonatum verticillatum (L.) All. & JAB-GUH-20564 & $V u^{3}$ & $\mathrm{H}$ & (Root $^{5}$ paste \& powder) $)^{2}$ & (Gastric problems5, wounds) $^{2}$ \\
\hline Primula denticulata Sm. & JAB-GUH-20606 & & $\mathrm{H}$ & $(\text { Flower \& root paste) })^{2}$ & 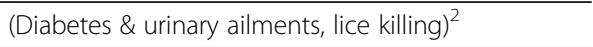 \\
\hline Prinsepia utilis Royle & JAB-GUH-20413 & & $S$ & $\left(\right.$ Seed $^{5}$ oil $^{2} \&\left(\text { Root }^{5} \text {-bark }\right)^{2}$ & $\begin{array}{l}\text { (Rheumatic pain, Diarrhoea) })^{2} \&(\text { Pile, Stomach } \\
\text { disorders) }\end{array}$ \\
\hline Pyrus pashia Buch.Ham. ex D. Don & JAB- GUH-20699 & & $\mathrm{T}$ & Fruits $^{2,4,5} \&$ Bark $^{4}$ & $\begin{array}{l}\text { (Digestive disorder) }^{2,5} \text { (Astringent, Laxative, } \\
\text { Anthelmintic, Febrifuge) }^{4}\end{array}$ \\
\hline Ranunculus hirtellus Royle. & JAB-GUH-20620 & & $\mathrm{H}$ & Plant paste ${ }^{2}$ & Wounds $^{2}$ \\
\hline Reinwardtia indica Dumort. & JAB-GUH-20566 & & $\mathrm{H}$ & Flowers $^{2}$ & Tongue wash ${ }^{2}$ \\
\hline Rhamnus virgatus Roxb. & JAB-GUH-20624 & & $\mathrm{S}$ & Bark paste \& Fruits $^{2}$ & Eczema \& Ring Worm, Emetic \& Purgative ${ }^{2}$ \\
\hline Rhododendron arboreum Smith & JAB-GUH-20521 & & $\mathrm{T}$ & Flower $^{2,4}$, Bark $^{2}, \&$ (Young Shoots) $)^{5}$ & $\begin{array}{l}\text { (Digestive and respiratory disorder) })^{2} \text { (tonic for heart, } \\
\text { diarrhoea \& dysentery) }{ }^{4} \text { (Headache, Blood } \\
\text { dysentery) }\end{array}$ \\
\hline Rhus javanica L. & JAB-GUH-20424 & & $\mathrm{S}$ & Fruits \& Bark Paste ${ }^{2}$ & Colic \& Cholera, Swelling \& Wounds ${ }^{2}$ \\
\hline Rosa sericea Lindl. & JAB-GUH-20626 & $R^{2}$ & S & Flower juice $^{2} \&$ Fruits $^{5}$ & Bowel complaints $^{2}$, (Headaches \& Liver complaints) $)^{5}$ \\
\hline Roscoea alpina Royle & JAB-BSD-114063 & $R^{2}$ & $\mathrm{H}$ & 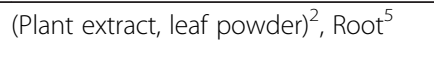 & 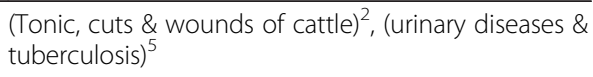 \\
\hline Roylea cinerea (D.Don) Baill. & JAB-GUH-20556 & & $\mathrm{S}$ & Leaves decoction ${ }^{2}$ & Malarial fever ${ }^{2}$ \\
\hline Rubia manjith Roxb. ex Fleming & JAB-GUH-20647 & & S & (Roots $^{5} \&$ Flowers $)^{2}$ & $\begin{array}{l}\text { (Tonic \& Astringent, Bacillary Dysentery) })^{2} \text {, (Lower } \\
\text { blood pressure, Kidney stone) }\end{array}$ \\
\hline Rubus nepalensis (Hook.f.) Kuntze & JAB-GUH-20625 & & $\mathrm{H}$ & Root paste ${ }^{2}$ & Burns \& scalds ${ }^{2}$ \\
\hline Rubus niveus Thunb & JAB-GUH-20638 & & $\mathrm{S}$ & Fruit extract \& Fruit juice ${ }^{2}$ & Dysmenorrhoea \& Antidote of snake bite ${ }^{2}$ \\
\hline Rumex hastatus D.Don & JAB-GUH-20603 & & $\mathrm{H}$ & Leaf extract $^{2}$ & ${\text { (Cuts \& wounds, nettle sitting reliever) }{ }^{2}}^{2}$ \\
\hline Rumex nepalensis Spreng. & JAB-GUH-20602 & & $\mathrm{H}$ & Leaf $^{5}$ infusion ${ }^{2}$ & ${\text { (Dysmenorrhoea, stomach ache) })^{2} \text {, Etching }}^{5}$ \\
\hline Salvia hians Royle ex Benth. & JAB-GUH-20555 & $R^{2}$ & $\mathrm{H}$ & Leaf juice $^{2}$, Root $^{5}$ & $\begin{array}{l}\text { (Arthritic, pain \& eczema, body swelling)2, (cold, } \\
\text { coughs \& anxiety) }\end{array}$ \\
\hline Salvia nubicola Wall. ex Sw. & JAB-GUH-20560 & & $\mathrm{H}$ & ${\left.\text { (Leaf paste, } \text { Root }^{5} \text { extract }\right)^{2}}^{2}$ & (Wounds, cold \& cough) $^{2}$, Fever ${ }^{5}$ \\
\hline Sapindus mukorossi Gaertn. & JAB-GUH-20649 & & $\mathrm{T}$ & Fruit $^{2,4} \&$ Seed $^{4}$ & $\begin{array}{l}\text { Hair }{ }^{2} \text { (Expectorant, antiepileptic, Emetic, febrifuge \& } \\
\text { Dental cares) }{ }^{4}\end{array}$ \\
\hline Sarcococca saligna (D.Don) Muell.-Arg. & JAB-GUH-20486 & & S & Leaves $^{2}$, Roots ${ }^{5}$ & Joint pain ${ }^{2}$, Bawseer $^{5}$ \\
\hline Saussurea albescens (DC.) Sch.-Bip. & JAB-GUH-20466 & & $\mathrm{H}$ & Flower heads $^{2}$ & Bronchitis reliever $^{2}$ \\
\hline Saussurea auriculata (Spreng. ex DC.) Sch.-Bip. & JAB-BSD-114072 & $R^{2}$ & $\mathrm{H}$ & Leaf paste $^{2}$ & Venereal diseases $^{2}$ \\
\hline Saxifraga diversifolia Wall. ex Ser. & JAB-BSD-14071 & & $\mathrm{H}$ & Root extract $^{2}$ & Vermifuge $^{2}$ \\
\hline Selinum candollii DC. & JAB-GUH-20409 & & $\mathrm{H}$ & Root $^{5}$ powder $^{2}$ & 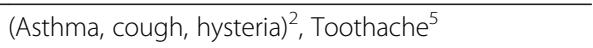 \\
\hline Senecio graciliflorus DC. & JAB-GUH-20462 & & $\mathrm{H}$ & (Leaf paste \& Juice of heads) $^{2}$ & ${\text { (Ringworm diseases \& insect bites, pussed ear) }{ }^{2}}^{2}$ \\
\hline Silene edgeworthii Bocquet. & JAB-GUH-20499 & & $\mathrm{H}$ & $(\text { Leaf \& young shoots juice })^{2}$ & Eye infections ${ }^{2}$ \\
\hline Solanum suratteuse Burm. & JAB-GUH-20660 & & $\mathrm{H}$ & (Fruits \& flower buds) $^{2}$ & (Fever, cough, asthama, gonorrhoea, eye ailments) $^{2}$ \\
\hline Solidago virgaurea $\mathrm{L}$. & JAB-BSD-114061 & & $\mathrm{H}$ & ${\text { (Leaves \& herb juice, Chewed roots) })^{2}}$ & $\begin{array}{l}\text { (Kidney troubles, asthma, rheumatism, wounds, } \\
\text { throat irritation)2 }\end{array}$ \\
\hline
\end{tabular}


Table 1 Plant species with their status and the part used in different ailments (Continued)

\begin{tabular}{|c|c|c|c|c|c|}
\hline Sorbaria tomentosa (Lindl.) Rehder & JAB-GUH-20637 & & $\bar{S}$ & Fruits (smoke) $)^{2}$ & Asthama $^{2}$ \\
\hline Sorbus aucuparia L. & JAB-GUH-20632 & $R^{2}$ & $\mathrm{~T}$ & Fruit extract ${ }^{2}$ & Cough \& Cold $^{2}$ \\
\hline Sorbus cuspidata (Spach) Hedlund & JAB-GUH-20634 & $R^{2}$ & $T$ & Bark decoction $^{2}$ & Fever $^{2}$ \\
\hline Swertia chirayita (Roxb. ex Fleming) Karsten & JAB-GUH-20538 & $E^{3}, V u^{6}$ & $\mathrm{H}$ & Leaves $^{5}$ & Blood diseases $^{5}$ \\
\hline Swertia ciliata (G.Don) Burtt. & JAB-BSD-114044 & & $\mathrm{H}$ & Plant extract ${ }^{2}$, Leaves ${ }^{5}$ & Malaria $^{2}$, Blood purifier ${ }^{5}$ \\
\hline Synotis alatus (Wall. ex DC.) C. Jeffrey \& Chen. & JAB-GUH-20681 & $R^{2}$ & $\mathrm{H}$ & Plant decoction ${ }^{2}$ & Fever $^{2}$ \\
\hline Taraxacum officinale Weber. & JAB-GUH-20465 & & $\mathrm{H}$ & Root $^{5}$ extract ${ }^{2}$ & 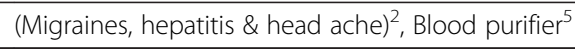 \\
\hline Taxus baccata $\mathrm{L}$. & JAB-GUH-20661 & & $T$ & Bark $^{2,5} \&$ Bark Paste $^{2}$ & $\begin{array}{l}\text { (Plaster on fractured bones Headache })^{2} \&(\text { Breast } \\
\text { Pile })^{5}\end{array}$ \\
\hline Triumfetta rhomboidea Jacq. & JAB-GUH-20662 & & $\mathrm{H}$ & (Root juice, Fruits \& Leaves) ${ }^{2}$ & ${\text { (Cuts, delivery facilitation) })^{2}}^{2}$ \\
\hline Urena lobata L. & JAB-GUH-20568 & & $\mathrm{H}$ & Root paste $^{2}$ & (Body pain \& rheumatism)2 \\
\hline Urtica ardens Link. & JAB-GUH-20668 & & $\mathrm{H}$ & (Seed oil \& Leaf extract) $^{2}$ & $\begin{array}{l}\text { (Sciatica, rheumatism, skin ailments, hair-wash for } \\
\text { avoiding baldness) }\end{array}$ \\
\hline Urtica dioica $\mathrm{L}$. & JAB-GUH-20664 & & $\mathrm{H}$ & (Seed oil \& Leaf extract) $)^{2}$, Root $^{5}$ & $\begin{array}{l}\text { (Sciatica, rheumatism, skin ailments, hair-wash for } \\
\text { avoiding baldness) })^{2}, \text { Boils }\end{array}$ \\
\hline Valeriana hardwickii Wallich & JAB-GUH-20671 & & $\mathrm{H}$ & (Root $^{5}$ decoction \& Root paste) $)^{2}$ & (Urinary disorder, joint pains) $^{2}$, Wounds $^{5}$ \\
\hline Verbascum thapsus L. & JAB-GUH-20652 & & $\mathrm{H}$ & 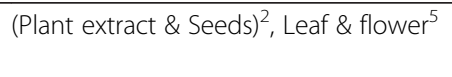 & $\begin{array}{l}\text { (Asthma, bronchitis, narcotic) })^{2} \text { (Ulcers, tumors \& } \\
\text { piles) }\end{array}$ \\
\hline Vernonia anthelmintica (L.) Willd. & JAB-GUH-20455 & & $\mathrm{H}$ & Leaf powder ${ }^{2}$ & $\left(\right.$ Intestinal disorder, fever \& skin ailments) ${ }^{2}$ \\
\hline Vernonia cinerea (L.) Less. & JAB-GUH-20407 & & $\mathrm{H}$ & $(\text { Leaf extract \& seeds) })^{2}$ & (Dysentery, cold \& cough) ${ }^{2}$ \\
\hline Veronica anagallis-aquatica Linn. & JAB-GUH-20658 & & $\mathrm{H}$ & Plant juice $^{2}$ & (Cuts, burns \& sores) $^{2}$ \\
\hline Viburnum cotinifolium D.Don & JAB-GUH-20496 & & $\mathrm{T}$ & Bark decoction $^{2}$ & Hepatic \& digestive disorder ${ }^{2}$ \\
\hline Viburnum erubescens Wall. ex DC. & JAB-GUH-20490 & & $\mathrm{S}$ & Leaves $^{2}$ & Insecticide $^{2}$ \\
\hline Viburnum grandiflorum Wall ex DC. & JAB-GUH-20492 & & $\mathrm{S}$ & Bark Decoction $^{2}$ & Hepatic troubles $^{2}$ \\
\hline Viburnum nervosum D.Don & JAB-GUH-20493 & & $\mathrm{S}$ & Bark Decoction $^{2}$ & Menorrhagia $^{2}$ \\
\hline Viola canescens Wallich & JAB-GUH-20690 & & $\mathrm{H}$ & (Plant $^{4}$ decoction, Root \& Leaf ${ }^{5}$ juice $)^{2}$ & $\begin{array}{l}\text { (Malarial fever, bronchitis, asthma, emetic, cuts \& } \\
\text { wounds) }{ }^{2} \text {, (Headache,cold, cough \& malaria) } \\
\text { (Expectorant, antipyretic, diaphoretic) }\end{array}$ \\
\hline Woodfordia fruticosa (L.) Kurz & JAB-GUH-20567 & & $\mathrm{S}$ & Leaves $\&$ bark, Dry flowers $^{2}$ & Febrifuge, Haemorrhoids ${ }^{2}$ \\
\hline Zanthoxylum armatum DC & JAB-GUH-20460 & $V u^{3}$ & $\mathrm{~S}$ & Leaves \& Fruits $^{2}(\text { Seed \& Bark) })^{5}$ & $\begin{array}{l}\text { Mouth wash }{ }^{2} \& \text { tooth ache } e^{2,5} \text { (Infection in stored } \\
\text { grain) })^{5}\end{array}$ \\
\hline
\end{tabular}

Board $\left(\right.$ NMPB, 2003), ${ }^{(4)}=$ Joshi et al. 2010, ${ }^{(5)}=$ Singh \& Rawat 2011, ${ }^{(6)}=$ Semwal et al. 2007.) J.A.B = Jahangeer Akbar Bhat, GUH = Garhwal University Herbarium, BSD $=$ Botanical Survey Dehradun. 


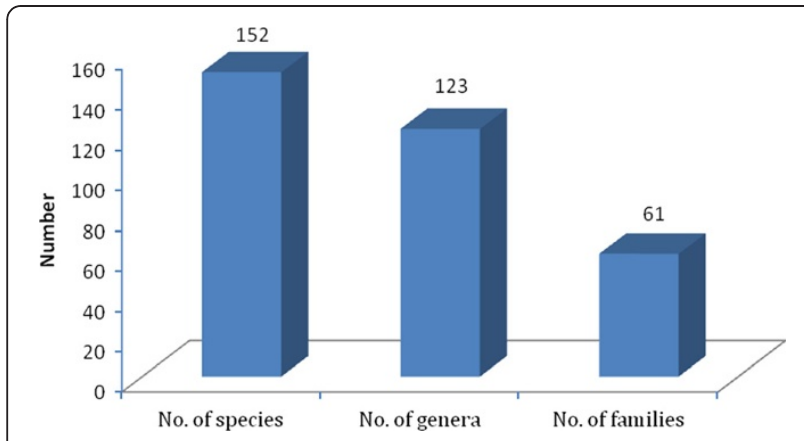

Figure 2 Total numbers of species, genera and families of plants having medicinal values.

andomly from the villages. Gundhaar is situated inside the sanctuary with 42 households while village Ransi is situated at the fringe of sanctuary with a total of 119 households. More than 10 percent of respondents of total population of the villages were selected for questionnaire survey. Both formal and informal discussions were carried out covering different age groups with both genders and mostly elders were involved in the interview process. The plants reported in ecological studies were also further used to collect the informations on ethnomedicinal uses with relevant available literature in Himalayan region and in a part of Kedarnath Wildlife Sanctuary [19,27,28]. The plant species having ethnomedicinal values were cross checked with the Red Data Book and other publications who have categorized the plant species under various threat (ecological status) categories [29-31].

\section{Collection and identification of plant specimens}

From each sampling site, all plant species encountered in the quadrats were collected, and identified with the help of local and regional floras [27,32], while as some plants were also identified with the field guide [33]. Specimens collected during the surveys were processed in the laboratory according to [34]. These were pressed, dried in blotting sheets and poisoned with formaldehyde or mercuric chloride solution $(0.5 \%)$ to protect against insect and fungal damage before mounting on the herbarium sheets. Voucher specimens were deposited in the Herbarium of Botanical Survey of India (BSD) and in the Herbarium of HNB Garhwal University Srinagar (GUH) under collector series JAB (Jahangeer Akbar Bhat). The nomenclature of the species follows "Flowering Plants of Uttarakhand - A Checklist" [35].

\section{Results and discussion}

A total of one hundred and fifty two species of medicinally important plants (Table 1) were found in the quadrats including 49 species (Table 5) reported from the villages Gundhaar and Ransi of Madhmeshwar area. One hundred and three of these were herbs, thirty two shrubs and seventeen trees (Table 1), belonging to hundred twenty three genera of sixty one families (Figure 2). The most commonly used parts of ethomedicinal plants, compiled with relevant literature were leaves (32\%), roots $(24 \%)$, whole plants or plant $(13 \%)$, followed by fruits $(9 \%)$ and seeds and flowers (6\% each) (Figure 3 and Table 2). According to different reports [19,27-31] eighteen plant species encountered have to be classified as rare, endangered, critically endangered or vulnerable (Table 2): Aconitium hetrophyllum, Picrorhiza kurrooa, Podophyllum hexandrum, Rosa sericea, Roscoea alpina, Salvia hians, Saussurea auriculata, Sorbus aucuparia, Sorbus cuspidata, Synotis alatus, Bistorta amlexicaulis, Coriaria nepalensis, Hypericum choisianum and Morina

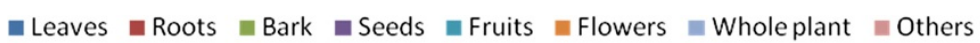

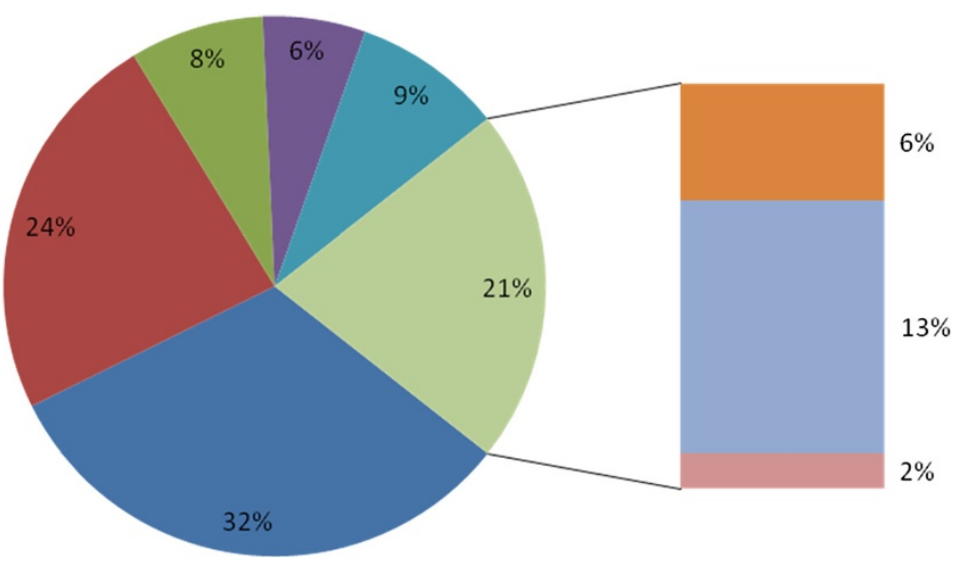

Figure 3 Percentage of plant parts used in preparing medicines for various ailments. 
Table 2 Medicinal tree species in the study area (F- Frequency \%, D- Density trees/100 $\mathrm{m}^{2}$ )

\begin{tabular}{|c|c|c|c|c|c|c|c|c|c|c|c|}
\hline \multirow[t]{2}{*}{ Species } & \multirow[t]{2}{*}{ Family } & \multicolumn{2}{|c|}{$\begin{array}{c}\text { Zone-I } \\
(1550-1750 \mathrm{~m})\end{array}$} & \multicolumn{2}{|c|}{$\begin{array}{c}\text { Zone-II } \\
(2000-2200 \mathrm{~m})\end{array}$} & \multicolumn{2}{|c|}{$\begin{array}{c}\text { Zone-III } \\
(2450-2650 \mathrm{~m})\end{array}$} & \multicolumn{2}{|c|}{$\begin{array}{c}\text { Zone-IV } \\
(2900-3100 \mathrm{~m})\end{array}$} & \multicolumn{2}{|c|}{$\begin{array}{c}\text { Zone-V } \\
(3350-3550 \mathrm{~m})\end{array}$} \\
\hline & & $F$ & D & $\mathbf{F}$ & D & $\mathbf{F}$ & D & $\mathrm{F}$ & D & $\mathbf{F}$ & D \\
\hline Abies pindrow & Pinaceae & - & - & - & - & - & - & 20 & 0.25 & 10 & 0.10 \\
\hline Aesculus indica & Hippocastanaceae & 15 & 0.15 & - & - & - & - & - & - & - & - \\
\hline Callicarpa arborea & Verbenaceae & 5 & 0.05 & - & - & - & - & - & - & - & - \\
\hline Carpinus viminea & Corylaceae & - & - & 5 & 0.10 & - & - & - & - & - & - \\
\hline Hippophae salicifolia & Elaegnaceae & - & - & - & - & 10 & 0.15 & - & - & - & - \\
\hline Juglans regia & Juglandaceae & 5 & 0.15 & 5 & 0.05 & - & - & - & - & - & - \\
\hline Lyonia ovalifolia & Ericaceae & 25 & 0.30 & 25 & 0.50 & 15 & 0.30 & - & - & - & - \\
\hline Myrica esculenta & Myricaceae & 20 & 0.30 & - & - & - & - & - & - & - & - \\
\hline Neolitsea pallens & Lauraceae & 30 & 0.45 & 5 & 0.05 & - & - & - & - & - & - \\
\hline Pinus roxburghii & Pinaceae & 35 & 0.55 & - & - & - & - & - & - & - & - \\
\hline Pyrus pashia & Rosaceae & 10 & 0.10 & - & - & - & - & - & - & - & - \\
\hline Rhododendron arboreum & Ericaceae & 25 & 0.25 & 50 & 0.90 & 50 & 1.10 & - & - & - & - \\
\hline Sapindus mukorossi & Sapindaceae & 10 & 0.15 & - & - & - & - & - & - & - & - \\
\hline Sorbus aucuparia & Rosaceae & - & - & - & - & - & - & - & - & 25 & 0.25 \\
\hline Sorbus cuspidata & Rosaceae & - & - & - & - & 15 & 0.15 & - & - & - & - \\
\hline Taxus baccata & Taxaceae & - & - & - & - & - & - & 10 & 0.10 & - & - \\
\hline Viburnum cotinifolium & Caprifoliaceae & - & - & - & - & 15 & 0.25 & 25 & 0.35 & - & - \\
\hline
\end{tabular}

longifolia were recorded as rare species, while Jurinea dolomiaea and Swertia chirayita are classified as endangered, and Polygonatum verticillatum and Zanthoxylum armatum are vulnerable (Table 1 ).

\section{Ecological study of plant species Altitudinal zone-I (1550-1750 m)}

In this altitudinal zone ten trees were reported having medicinal values. The highest density $\left(0.55\right.$ trees $\left./ 100 \mathrm{~m}^{2}\right)$ and frequency (35\%) was found for Pinus roxburghii followed by Neolitsea pallens ( 0.45 trees/100 $\mathrm{m}^{2}$ density with $30 \%$ frequency). The lowest density (0.05 trees/ $100 \mathrm{~m}^{2}$ ) and frequency (5\%) was observed for Callicarpa arborea (Table 2). Sixteen medicinal shrub species were found in this altitudinal zone. The highest density and frequency (1.85 plants/25 $\mathrm{m}^{2}$ and $52.50 \%$ respectively) was recorded for Debregeasia salicifolia followed by Woodfordia fruticosa (0.58 plants/25 $\mathrm{m}^{2}$, frequency 17.50\%). Arachne cordifolia and Sarcococca saligna were found with the lowest density and frequency (0.18 plants/ $25 \mathrm{~m}^{2}, 7.50 \%$ ) (Table 3). A total of twenty nine herb species with medicinal values were found. Among the herb species, the highest density $\left(0.93\right.$ plants $\left./ \mathrm{m}^{2}\right)$ was observed for Gonatanthus pumilus followed by Rumex hastatus (0.69 plants $\left./ \mathrm{m}^{2}\right)$. The highest frequency was again reported for Gonatanthus pumilus (16.25\%) followed by Aster peduncularis and Cyathula tomentosa (15\%). The lowest density and frequency (0.05 plants $/ \mathrm{m}^{2}$ and $1.25 \%$ respectively) was recorded for Valeriana hardwickii (Table 4).

\section{Altitudinal zone-II (2000-2200 m)}

In this altitudinal zone-II, five trees, eight shrubs and twenty nine herbs with medicinal values were observed (Table 2, Table 3 and Table 4 respectively). The highest density $\left(0.90\right.$ trees $\left./ 100 \mathrm{~m}^{2}\right)$ and frequency (50\%) was found for Rhododendron arboreum followed by Lyonia ovalifolia (0.50 trees/100 $\left.\mathrm{m}^{2}, 25 \%\right)$. The lowest density (0.05 trees/ $100 \mathrm{~m}^{2}$ ) and frequency (5\%) was observed for both Juglans regia and Neolitsea pallens (Table 2). The highest density and frequency for shrubs (0.85 plants $\left./ 25 \mathrm{~m}^{2}, 17.50 \%\right)$ was recorded for Rubus niveus, followed by Indigofera heterantha (0.68 plants $\left./ 25 \mathrm{~m}^{2}, 15 \%\right)$ values. The lowest density (0.15 plants $\left./ 25 \mathrm{~m}^{2}, 2.50 \%\right)$ was reported for Sarcococca saligna (Table 3 ). A total of twenty nine herbs were found with medicinal values and the highest density (1.30 plants $/ \mathrm{m}^{2}$ ) and frequency $(32.50 \%)$ was observed for Ainsliaea latifolia, followed by Rumex nepalensis (1.13 plants $\left./ \mathrm{m}^{2}, 26.25 \%\right)$. The lowest density (0.05 plants $/ \mathrm{m}^{2}$ ) was reported for Asparagus filicinus, Paeonia emodii, Verbascum thapsus, Bergenia ciliata, and Reinwardtia indica $\left(0.08\right.$ plants $\left./ \mathrm{m}^{2}\right)$. The lowest frequency (1.25\%) was recorded for Paeonia emodii (Table 4).

\section{Altitudinal zone-III (2450-2650 m)}

In this altitudinal zone-III, five trees with medicinal values were reported. Among these medicinal tree species, the 
Table 3 Medicinal shrub species in the study area (F- Frequency \%, D- Density plants/25 $\mathrm{m}^{2}$ )

\begin{tabular}{|c|c|c|c|c|c|c|c|c|c|c|c|}
\hline \multirow[t]{2}{*}{ Species } & \multirow[t]{2}{*}{ Family } & \multicolumn{2}{|c|}{$\begin{array}{c}\text { Zone-I } \\
(1550-1750 \mathrm{~m})\end{array}$} & \multicolumn{2}{|c|}{$\begin{array}{c}\text { Zone-II } \\
(2000-2200 \mathrm{~m})\end{array}$} & \multicolumn{2}{|c|}{$\begin{array}{c}\text { Zone-III } \\
(2450-2650 \mathrm{~m})\end{array}$} & \multicolumn{2}{|c|}{$\begin{array}{c}\text { Zone-IV } \\
(2900-3100 \mathrm{~m})\end{array}$} & \multicolumn{2}{|c|}{$\begin{array}{c}\text { Zone-V } \\
(3350-3550 \mathrm{~m})\end{array}$} \\
\hline & & $\mathbf{F}$ & D & $\mathbf{F}$ & D & $\mathbf{F}$ & D & $\mathbf{F}$ & D & $\mathrm{F}$ & D \\
\hline Arachne cordifolia & Euphorbiaceae & 10.00 & 0.18 & - & - & - & - & - & - & - & - \\
\hline Buddleja asiatica. & Buddlejaceae & 12.50 & 0.43 & - & - & - & - & - & - & - & - \\
\hline Clematis buchananiana & Ranunculaceae & - & - & - & - & 5.00 & 0.20 & - & - & - & - \\
\hline Coriaria nepalensis & Coriariaceae & - & - & 12.50 & 0.25 & 10.00 & 0.58 & 70.00 & 2.73 & - & - \\
\hline Cotoneaster microphyllus & Rosaceae & - & - & - & - & - & - & 17.50 & 0.68 & 33.33 & 0.23 \\
\hline Debregeasia salicifolia & Urticaceae & 52.50 & 1.85 & 7.50 & 0.35 & - & - & - & - & - & - \\
\hline Desmodium elagans & Fabaceae & - & - & 7.50 & 0.20 & 5.00 & 0.18 & - & - & - & - \\
\hline Deutzia compacta & Hydrangeaceae & - & - & - & - & 7.50 & 0.30 & - & - & - & - \\
\hline Elsholtzia fruticosa & Lamiaceae & - & - & 10.00 & 0.25 & 12.50 & 0.25 & - & - & - & - \\
\hline Eupatorium odenophorum & Asteraceae & 12.50 & 0.48 & - & - & - & - & - & - & - & - \\
\hline Holmskioldia sanguinea & Verbenaceae & 10.00 & 0.38 & - & - & - & - & - & - & - & - \\
\hline Hypericum choisianum & Hyperiaceae & 10.00 & 0.20 & 5.00 & 0.18 & - & - & - & - & - & - \\
\hline Indigofera heterantha & Fabaceae & 12.50 & 0.35 & 15.00 & 0.68 & 40.00 & 1.53 & - & - & - & - \\
\hline Inula cappa & Asteraceae & - & - & - & - & 12.50 & 0.40 & - & - & - & - \\
\hline Leptodermis lanceolata & Rubiaceae & 15.00 & 0.43 & - & - & - & - & - & - & - & - \\
\hline Leycesteria formosa & Caprifoliaceae & - & - & - & - & 5.00 & 0.15 & 7.50 & 0.38 & - & - \\
\hline Lonicera angustifolia & Caprifoliaceae & - & - & - & - & - & - & 20.00 & 0.48 & 33.33 & 0.38 \\
\hline Persicaria polystachya & Polygonaceae & - & - & - & - & 2.50 & 0.05 & 7.50 & 0.35 & - & - \\
\hline Prinsepia utilis & Rosaceae & 10.00 & 0.25 & - & - & - & - & - & - & - & - \\
\hline Rhamnus virgatus & Rhamnaceae & 15.00 & 0.33 & - & - & - & - & - & - & - & - \\
\hline Rhus javanica & Anacardiaceae & 10.00 & 0.33 & - & - & - & - & - & - & - & - \\
\hline Rosa sericea & Rosaceae & - & - & - & - & 12.50 & 0.65 & 40.00 & 1.63 & 26.53 & 1.23 \\
\hline Roylea cinerea & Lamiaceae & 15.00 & 0.33 & - & - & - & - & - & - & - & - \\
\hline Rubia manjith & Rubiaceae & - & - & - & - & 10.00 & 0.28 & - & - & - & - \\
\hline Rubus niveus & Rosaceae & 10.00 & 0.28 & 17.50 & 0.85 & 35.00 & 1.45 & - & - & - & - \\
\hline Sarcococca saligna & Buxaceae & 7.50 & 0.23 & 2.50 & 0.15 & - & - & - & - & - & - \\
\hline Sorbaria tomentosa & Rosaceae & - & - & - & - & 12.50 & 0.28 & - & - & - & - \\
\hline Viburnum erubescens & Caprifoliacae & - & - & - & - & 12.50 & 0.40 & 10.00 & 0.75 & - & - \\
\hline Viburnum grandiflorum & Caprifoliacae & - & - & - & - & 15.00 & 0.43 & 32.50 & 1.05 & - & - \\
\hline Viburnum nervosum & Caprifoliacae & - & - & - & - & - & - & 12.50 & 0.38 & - & - \\
\hline Woodfordia fruticosa & Lythraceae & 17.50 & 0.58 & - & - & - & - & - & - & - & - \\
\hline Zanthoxylum armatum & Rutaceae & 10.00 & 0.33 & - & - & - & - & - & - & - & - \\
\hline
\end{tabular}

highest density $\left(1.10\right.$ trees/100 $\left.\mathrm{m}^{2}\right)$ and frequency (50\%) was observed for Rhododendron arboreum. The lowest density $\left(0.15\right.$ trees $\left./ 100 \mathrm{~m}^{2}\right)$ was recorded for Hippophae salicifolia and Sorbus cuspidata while as lowest frequency (10\%) was observed for Hippophae salicifolia (Table 2). Fifteen shrub species with medicinal values were found in this altitudinal zone. The highest density and frequency (1.53 plants $/ 25 \mathrm{~m}^{2}$ and $40 \%$ ) was recorded for Indigofera heterantha followed by Rubus niveus (1.45 plants $/ 25 \mathrm{~m}^{2}$, $35 \%)$, while the lowest density and frequency ( 0.05 plants/ $25 \mathrm{~m}^{2}, 2.50 \%$ ) was registered for Persicaria polystachya (Table 3). In the herb layer thirty (30) species were found.
Among these Rumex nepalensis had the highest density and frequency $\left(0.66\right.$ plants $\left./ \mathrm{m}^{2}, 23.75 \%\right)$, followed by Veronica anagallis-aquatica (0.61 plants $\left./ \mathrm{m}^{2}, 18.75 \%\right)$. The lowest density $\left(0.06\right.$ plants $\left./ \mathrm{m}^{2}\right)$ was recorded for Pimpinella acuminata (Table 4).

\section{Altitudinal zone-IV (2900-3100 m)}

In this altitudinal zone-IV, three tree species, nine shrub species and twenty two herb species with medicinal values were encountered (Table 2, Table 3 and Table 4). In the tree layer, the highest density $\left(0.35\right.$ trees $\left./ 100 \mathrm{~m}^{2}\right)$ and frequency $(25 \%)$ was found for Viburnum cotnifolium 
Table 4 Medicinal herb species in the study area (F- Frequency \%, D- Density plants $/ \mathrm{m}^{2}$ )

\begin{tabular}{|c|c|c|c|c|c|c|c|c|c|c|c|}
\hline \multirow[t]{2}{*}{ Species } & \multirow[t]{2}{*}{ Family } & \multicolumn{2}{|c|}{$\begin{array}{c}\text { Zone-I } \\
(1550-1750 \mathrm{~m})\end{array}$} & \multicolumn{2}{|c|}{$\begin{array}{c}\text { Zone-II } \\
(2000-2200 \mathrm{~m})\end{array}$} & \multicolumn{2}{|c|}{$\begin{array}{c}\text { Zone-III } \\
(2450-2650 \mathrm{~m})\end{array}$} & \multicolumn{2}{|c|}{$\begin{array}{c}\text { Zone-IV } \\
(2900-3100 \mathrm{~m})\end{array}$} & \multicolumn{2}{|c|}{$\begin{array}{c}\text { Zone-V } \\
(3350-3550 \mathrm{~m})\end{array}$} \\
\hline & & $\mathrm{F}$ & D & $\mathbf{F}$ & D & $\mathrm{F}$ & D & $\mathrm{F}$ & D & $F$ & D \\
\hline Aconitium hetrophyllum & Ranunculaceae & - & - & - & - & - & - & - & - & 18.75 & 0.33 \\
\hline Ainsliaea apetra & Asteraceae & - & - & - & - & - & - & 31.25 & 0.95 & - & - \\
\hline Ainsliaea latifolia & Asteraceae & - & - & 32.50 & 1.30 & 11.25 & 0.16 & - & - & - & - \\
\hline Anaphalis contorta & Asteraceae & - & - & - & - & 10.00 & 0.20 & 23.75 & 0.69 & - & - \\
\hline Anaphalis margaritaceae & Asteraceae & - & - & - & - & 6.25 & 0.16 & 10.00 & 0.16 & - & - \\
\hline Anaphalis triplinervis & Asteraceae & 6.25 & 0.21 & 8.75 & 0.20 & - & - & - & - & - & - \\
\hline Anemone obtusiloba & Ranunculaceae & - & - & - & - & - & - & - & - & 15.00 & 0.26 \\
\hline Anemone rivularis & Ranunculaceae & - & - & 3.75 & 0.10 & 11.25 & 0.26 & - & - & - & - \\
\hline Arisaema jacquemontii & Araceae & - & - & - & - & 8.75 & 0.23 & 11.25 & 0.29 & - & - \\
\hline Artemisia japonica & Asteraceae & - & - & 13.75 & 0.34 & - & - & - & - & - & - \\
\hline Artemisia roxburghiana & Asteraceae & - & - & - & - & 7.50 & 0.26 & 2.50 & 0.05 & - & - \\
\hline Asparagus filicinus & Asparagaceae & - & - & 3.75 & 0.05 & 10.00 & 0.33 & - & - & - & - \\
\hline Aster peduncularis & Asteraceae & 15.00 & 0.30 & - & - & - & - & - & - & - & - \\
\hline Barleria cristata & Acanthaceae & 10.00 & 0.25 & - & - & - & - & - & - & - & - \\
\hline Begonia picta & Begoniaceae & - & - & - & - & - & - & 8.75 & 0.21 & - & - \\
\hline Bergenia ciliate & Saxifragaceae & - & - & 3.75 & 0.08 & 10.00 & 0.18 & 3.75 & 0.09 & - & - \\
\hline Bidens bipinnata & Asteraceae & - & - & 6.25 & 0.15 & - & - & - & - & - & - \\
\hline Bidens biternata & Asteraceae & 5.00 & 0.20 & - & - & - & - & - & - & - & - \\
\hline Bidens pilosa & Asteraceae & - & - & 12.50 & 0.24 & - & - & - & - & - & - \\
\hline Bistorta amplexicaulis & Polygonaceae & - & - & - & - & 10.00 & 0.36 & 21.25 & 0.61 & 2.50 & 0.21 \\
\hline Bistorta vaccinifolia & Polygonaceae & - & - & - & - & - & - & - & - & 7.50 & 0.13 \\
\hline Blumea lanceolaria & Asteraceae & 7.50 & 0.20 & - & - & - & - & - & - & - & - \\
\hline Bupleurum falcatum & Apiaceae & - & - & 8.75 & 0.18 & - & - & - & - & - & - \\
\hline Calanthe tricarinata & Orchidaceae & - & - & - & - & - & - & - & - & 11.25 & 0.20 \\
\hline Cannabis sativa & Cannabinaceae & - & - & 6.25 & 0.25 & - & - & - & - & - & - \\
\hline Clematis montana & Ranunculaceae & 11.25 & 0.33 & - & - & - & - & - & - & - & - \\
\hline Clinopodium umbrosum & Lamiaceae & 3.75 & 0.09 & 8.75 & 0.16 & - & - & - & - & - & - \\
\hline Corallodiscus lanuginosus & Gesneriaceae & - & - & - & - & 12.50 & 0.16 & - & - & - & - \\
\hline Cyathula capitata & Amaranthaceae & 2.50 & 0.06 & 7.50 & 0.24 & - & - & - & - & - & - \\
\hline Cyathula tomentosa & Amaranthaceae & 15.00 & 0.59 & - & - & - & - & - & - & - & - \\
\hline Cynoglossum glochidiatum & Boraginaceae & - & - & 8.75 & 0.21 & - & - & - & - & - & - \\
\hline Cynoglossum lanceolatum & Boraginaceae & 8.75 & 0.26 & - & - & - & - & - & - & - & - \\
\hline Delphinium vestitum & Ranunculaceae & - & - & - & - & - & - & - & - & 8.75 & 0.16 \\
\hline Dicliptera bupleuroides & Acanthaceae & - & - & 12.50 & 0.20 & - & - & - & - & - & - \\
\hline Dipsacus inermis & Dipsacaceae & - & - & - & - & 5.00 & 0.09 & - & - & - & - \\
\hline Elephantopus scaber & Asteraceae & 7.50 & 0.13 & - & - & - & - & - & - & - & - \\
\hline Elsholtzia strobilifera & Lamiaceae & - & - & - & - & - & - & 10.00 & 0.25 & 3.75 & 0.10 \\
\hline Euphorbia chamaesyce & Euphorbiaceae & 8.75 & 0.14 & - & - & - & - & - & - & - & - \\
\hline Euphorbia hypericifolia & Euphorbiaceae & - & - & - & - & - & - & - & - & 6.25 & 0.16 \\
\hline Euphorbia pilosa & Euphorbiaceae & 3.75 & 0.09 & - & - & 8.75 & 0.25 & 12.50 & 0.38 & - & - \\
\hline Fagopyrum dibotrys & Polygonaceae & - & - & 7.50 & 0.23 & 7.50 & 0.14 & - & - & - & - \\
\hline Fragaria nubicola & Rosaceae & 12.50 & 0.13 & 7.50 & 0.18 & 10.00 & 0.20 & - & - & - & - \\
\hline Galinsoga parviflora & Asteraceae & - & - & - & - & 8.75 & 0.18 & - & - & - & - \\
\hline Galium aparine & Rubiaceae & - & - & - & - & - & - & 8.75 & 0.20 & 6.25 & 0.13 \\
\hline Galium asperifolium & Rubiaceae & - & - & - & - & - & - & - & - & 3.75 & 0.10 \\
\hline
\end{tabular}


Table 4 Medicinal herb species in the study area (F- Frequency \%, D- Density plants $/ \mathbf{m}^{2}$ ) (Continued)

\begin{tabular}{|c|c|c|c|c|c|c|c|c|c|c|c|}
\hline Geranium wallichianum & Gerianiaceae & - & - & - & - & 8.75 & 0.26 & 5.00 & 0.15 & - & - \\
\hline Gerbera gossypina & Asteraceae & 11.25 & 0.21 & - & - & - & - & - & - & - & - \\
\hline Girardiana diversifolia & Urticaceae & 12.50 & 0.61 & 6.25 & 0.25 & - & - & - & - & - & - \\
\hline Gonatanthus pumilus & Araceae & 16.25 & 0.93 & - & - & - & - & - & - & - & - \\
\hline Gonostegia hirta & Urticaceae & 2.50 & 0.08 & - & - & - & - & - & & - & - \\
\hline Impatiens scabrida & Balsamaniceae & - & - & - & - & - & - & 3.75 & 0.09 & - & - \\
\hline Jurinea dolomiaea & Asteraceae & - & - & - & - & - & - & - & - & 5.00 & 0.08 \\
\hline Lamium album & Lamiaceae & - & - & 6.25 & 0.11 & - & - & - & - & - & - \\
\hline Leucas lanata & Lamiaceae & - & - & - & - & 7.50 & 0.18 & - & - & - & - \\
\hline Lindenbergia indica & Scrophulariaceae & - & - & - & - & 12.50 & 0.24 & - & - & - & - \\
\hline Maianthemum purpureum & Liliaceae & - & - & - & - & - & - & - & - & 3.75 & 0.05 \\
\hline Morina longifolia & Morinaceae & - & - & 3.75 & 0.13 & 3.75 & 0.15 & 6.25 & 0.11 & - & - \\
\hline Nepeta ciliaris & Lamiaceae & - & - & 11.25 & 0.16 & - & - & - & - & - & - \\
\hline Nomocharis oxypetala & Liliaceae & - & - & - & - & - & - & - & - & 11.25 & 0.13 \\
\hline Origanum vulgare & Lamiaceae & - & - & - & - & - & - & - & - & 3.75 & 0.09 \\
\hline Paeonia emodii & Paenoniaceae & 5.00 & 0.18 & 1.25 & 0.05 & - & - & - & - & - & - \\
\hline Parnassia nubicola & Saxifragaceae & - & - & - & - & - & - & 15.00 & 0.21 & - & - \\
\hline Pedicularis hoffmeisteri & Scrophulariaceae & - & - & - & - & - & - & - & - & 12.50 & 0.21 \\
\hline Phalaris minor & Poaceae & 6.25 & 0.33 & - & - & - & - & - & - & - & - \\
\hline Picrorrhiza kurrooa & Scrophulariaceae & - & - & - & - & - & - & - & - & 12.50 & 0.23 \\
\hline Pimpinella acuminata & Apiaceae & - & - & 12.50 & 0.15 & 5.00 & 0.06 & - & - & - & - \\
\hline Pimpinella diversifolia & Apiaceae & - & - & 7.50 & 0.13 & - & - & - & - & 8.75 & 0.19 \\
\hline Plantago depressa & Plantaginaceae & - & - & - & - & - & - & - & - & 10.00 & 0.16 \\
\hline Plantago himalaica & Plantaginaceae & - & - & - & - & - & - & - & - & 10.00 & 0.20 \\
\hline Podophyllum hexandrum & Podophyllaceae & - & - & - & - & - & - & - & - & 11.25 & 0.19 \\
\hline Polygonatum verticillatum & Liliaceae & - & - & - & - & 8.75 & 0.11 & - & - & - & - \\
\hline Primula denticulate & Primulaceae & - & - & - & - & - & - & - & - & 18.75 & 0.31 \\
\hline Ranunculus hirtellus & Ranunculaceae & - & - & - & - & - & - & - & - & 20.00 & 0.38 \\
\hline Reinwardtia indica & Linaceae & - & - & 5.00 & 0.08 & 7.50 & 0.10 & - & - & - & - \\
\hline Roscoea alpine & Zingiberaceae & - & - & - & - & - & - & 13.75 & 0.19 & - & - \\
\hline Rubus nepalensis & Rosaceae & - & - & - & - & - & - & 38.75 & 1.15 & 5.00 & 0.11 \\
\hline Rumex hastatus & Polygonaceae & 12.50 & 0.69 & - & - & - & - & - & - & - & - \\
\hline Rumex nepalensis & Polygonaceae & - & - & 26.25 & 1.13 & 23.75 & 0.66 & - & - & 27.50 & 0.71 \\
\hline Salvia hians & Lamiaceae & - & - & - & - & - & - & - & - & 12.50 & 0.21 \\
\hline Salvia nubicola & Lamiaceae & - & - & - & - & - & - & - & - & 3.75 & 0.06 \\
\hline Saussurea albescens & Asteraceae & - & - & 6.25 & 0.09 & 16.25 & 0.71 & - & - & - & - \\
\hline Saussurea auriculata & Asteraceae & - & - & - & - & - & - & - & - & 13.75 & 0.19 \\
\hline Saxifraga diversifolia & Saxifragaceae & - & - & - & - & - & - & 10.00 & 0.15 & - & - \\
\hline Selinum candollii & Apiaceae & - & - & - & - & - & - & - & - & 8.75 & 0.20 \\
\hline Senecio graciliflorus & Asteraceae & - & - & - & - & - & - & - & - & 8.75 & 0.15 \\
\hline Silene edgeworthii & Caryophyllaceae & - & - & - & - & 12.50 & 0.23 & 7.50 & 0.14 & - & - \\
\hline Solanum suratteuse & Solanaceae & 8.75 & 0.16 & - & - & - & - & - & - & - & - \\
\hline Solidago virgaurea & Asteraceae & - & - & - & - & 8.75 & 0.13 & - & - & - & - \\
\hline Swertia chirayita & Gentianaceae & - & - & - & - & - & - & 12.50 & 0.21 & - & - \\
\hline Swertia ciliate & Gentianaceae & - & - & - & - & - & - & - & - & 18.75 & 0.30 \\
\hline Synotis alatus & Asteraceae & - & - & - & - & 7.50 & 0.13 & - & - & - & - \\
\hline Taraxacum officinale & Asteraceae & - & - & - & - & - & - & - & - & 7.50 & 0.13 \\
\hline Triumfetta rhomboidea & Tiliaceae & 8.75 & 0.21 & - & - & - & - & - & - & - & - \\
\hline
\end{tabular}


Table 4 Medicinal herb species in the study area (F- Frequency \%, D- Density plants $/ \mathbf{m}^{2}$ ) (Continued)

\begin{tabular}{|c|c|c|c|c|c|c|c|c|c|c|c|}
\hline Urena lobata & Malvaceae & 6.25 & 0.15 & - & - & - & - & - & - & - & - \\
\hline Urtica ardens & Urticaceae & 2.50 & 0.20 & - & - & - & - & - & - & - & - \\
\hline Urtica dioica & Urticaceae & 7.50 & 0.49 & 6.25 & 0.25 & - & - & - & - & - & - \\
\hline Valeriana hardwickii & Valerianaceae & 1.25 & 0.05 & - & - & - & - & - & - & - & - \\
\hline Verbascum thapsus & Scrophulariaceae & 2.50 & 0.10 & 3.75 & 0.05 & 5.00 & 0.08 & 11.25 & 0.30 & - & - \\
\hline Vernonia anthelmintica & Asteraceae & - & - & - & - & 7.50 & 0.16 & - & - & - & - \\
\hline Vernonia cinerea & Asteraceae & - & - & 7.50 & 0.15 & - & - & - & - & - & - \\
\hline Veronica anagallis-aquatica & Scrophulariaceae & 2.50 & 0.10 & - & - & 18.75 & 0.61 & - & - & - & - \\
\hline Viola canescens & Violaceae & - & - & - & - & - & - & 10.00 & 0.19 & - & - \\
\hline
\end{tabular}

followed by Abies pindrow. The lowest density and frequency $\left(0.10\right.$ trees/100 $\left.\mathrm{m}^{2}, 10 \%\right)$ was observed for Taxus baccata (Table 2). For shrub species, the highest density and frequency (2.73 plants $/ 25 \mathrm{~m}^{2}, 70 \%$ ) was recorded for Coriaria nepalensis while the lowest density $(0.35$ plants $/ 25 \mathrm{~m}^{2}$ ) was recorded for Persicaria polystachya. Leycesteria formosa and Persicaria polystachya had the lowest frequency (7.50\%) (Table 3). Among the herb species, the highest density (1.15 plants $\left./ \mathrm{m}^{2}\right)$ and frequency (38.75\%) was observed for Rubus nepalensis followed by Ainsliaea apetra (0.95 plants $\left./ \mathrm{m}^{2}, 31.25 \%\right)$. The lowest density and frequency $\left(0.05\right.$ plants $/ \mathrm{m}^{2}, 2.50 \%$ respectively) was found for Artemisia roxburghiana, followed by Bergenia ciliata and Impatiens scabrida (Table 4).

\section{Altitudinal zone-V (3350-3550 m)}

Only two trees species with medicinal value were reported in the altitudinal zone-V. Sorbus aucuparia had the highest density and frequency $\left(0.25\right.$ trees $/ 100 \mathrm{~m}^{2}$, $25 \%$ ), while Abies pindrow followed ( 0.10 trees $/ 100 \mathrm{~m}^{2}$, $10 \%$ ) (Table 2). Of the three shrub species encountered Rosa sericea was most common (1.23 plants $\left./ 25 \mathrm{~m}^{2}\right)$, followed by Lonicera angustifolia (0.38 plants $\left./ 25 \mathrm{~m}^{2}\right)$, and Cotoneaster microphyllus ( 0.23 plants $\left./ 25 \mathrm{~m}^{2}\right)$. The highest frequency (33.33\%) was observed for both Cotoneaster microphyllus and Lonicera angustifolia, while Rosa sericea was much less frequent (26.53\%) (Table 3). Among the thirty one herbs Rumex nepalensis (0.71 plants $/ \mathrm{m}^{2}$, $27.50 \%)$, and Ranunculus hirtellus (0.38 plants $\left./ \mathrm{m}^{2}, 20 \%\right)$ had the highest density and frequency. The lowest density value $\left(0.05\right.$ plants $\left./ \mathrm{m}^{2}\right)$ was found for Maianthemum purpureum, while Bistorta amplexicaulis was observed with lowest frequency $(2.50 \%)$ (Table 4$)$.

\section{Ethnomedicinal study of plant species}

Of the total one hundred and fifty two species of ethnomedicinal plants complied for ethnomedicinal uses in the Himalayan region and Kedarnath Wildlife Sanctuary areas including 49 plant species of these were too reported from both the villages (Gundhaar and Ransi) of study area of Madhmeshwar, in KWLS. The scientific names, part used and ethnonomedicinal uses of these plants reported from the villages Gundhaar and Ransi is shown in Table 5. The reported 49 ethnomedicinal plants used to cure several ailments such as fever, cough, pain, wounds, cuts, insecticides, diarrhoea, dysentery, kidney problems, eye diseases, stop bleeding, abdomen pain, indigestion, antiseptic, healing foot cracks, mouth wash, blood diseases etc. The contribution of plant parts used by the inhabitants of Gundhaar and Ransi villages, was reported highest for roots (32\%), followed by leaves (27\%). Flowers, seeds and fruits contributed $8 \%$ for each and lowest contribution was reported for barks and resin of $3 \%$ and $1 \%$ respectively.

Ethnobotany explains the holistic relationships between plants and people [36]. Rapid global biodiversity loss is an issue of critical concern, with approximately 5000 species of animals and 25,00 species of plants currently listed as endangered, threatened, or at risk of overexploitation [37]. The Himalayan range is rich in endemic and medicinal plant diversity [38]. Uncontrolled developmental activities are causing a great loss to the biodiversity in the Indian Himalayan region, where medicinal plants in particular are declining at a very fast rate due to their over exploitation for trade [39], and it is believed that excessive anthropogenic activities are the main cause of decline in the population and availability of medicinal plants in the region $[38,40]$. There are many protected areas (PAs) across the Himalayan region but not a single PA has been specifically established to ensure the conservation of medicinal plants.

The plant species reported from the Madhmeshwar area of KWLS were one hundred and fifty two species having medicinally important value with one hundred twenty three genera belonging to sixty one families. In comparison [41] explored the Pindari area of Nanda Biosphere Reserve and reported 224 plant species with medicinal values. [42] recorded 701 species of medicinal plants of which 138 species were trees, 135 shrubs and 421 were herbs in various forest types of Uttarakhand. [43] presented a list of 41 medicinal plants with their medicinal uses and mode of application of Pauri Garhwal Himalaya. [44] reported 135 species having medicinal values from 
Table $\mathbf{5}$ Medicinal uses of plant species reported from the present study area

\begin{tabular}{|c|c|c|}
\hline \multirow[t]{2}{*}{ Scientific name } & \multicolumn{2}{|l|}{ Present study } \\
\hline & Plant part used & Medicinal uses \\
\hline Aconitium hetrophyllum Wallich & Root & Fever and cough \\
\hline Aesculus indica (Wall. ex Cambess.) Hook.f. & Seed & Rheumatic pain \\
\hline Anaphalis margaritaceae (L.) Benth & Leaves & Wounds and cuts \\
\hline Anemone rivularis Buch.-Ham. ex DC & Leaves & Wounds \\
\hline Artemisia japonica Thunb. & Leaves & Insecticide \\
\hline Asparagus filicinus Buch.-Ham. ex D. Don & Root & Diarrhoea and dysentery \\
\hline Barleria cristata $\mathrm{L}$. & Root & Wounds \\
\hline Bergenia ciliata (Haw.) Sternb. & Root & Fever, kidney calculi, diarrhoea \\
\hline Blumea lanceolaria (Roxb.) Druce & Leaves & Cuts \\
\hline Dicliptera bupleuroides Nees & Leaves & Skin diseases, cough, wounds \\
\hline Elsholtzia strobilifera Benth. & Whole plant & Wounds \\
\hline Eupatorium odenophorum Spreng. & Leaves & Skin diseases \\
\hline Galium aparine L. & Roots & Eye diseases and stop bleeding \\
\hline Geranium wallichianum D. Don ex Sweet & Root & Dysentery and cold \\
\hline Girardiana diversifolia (Link) Friis & Whole plant & Abdomen pain and indigestion \\
\hline Hippophae salicifolia D.Don & Fruits & Dandruff \\
\hline Indigofera heterantha Wall. ex Brandis & Leaves & Dysentery and cough \\
\hline Juglans regia $\mathrm{L}$. & Leaves & Insecticides \\
\hline Jurinea dolomiaea Boiss. & Root & Incense \\
\hline Leycesteria formosa Wallich & Leaves & Lice killing \\
\hline Morina longifolia Wall. ex DC. & Root & Antiseptic, Burns, wounds \\
\hline Myrica esculenta Buch.-Ham. ex D. Don & Leaves and fruits & Skin diseases and wounds \\
\hline Origanum vulgare $\mathrm{L}$. & Leaves & Tooth ache \\
\hline Pedicularis hoffmeisteri Klotz. & Whole plant & Indigestion \\
\hline Phalaris minor Retz. & Root & Wounds \\
\hline Picrorhiza kurrooa Royle ex Benth. & Root & Stomach ache \\
\hline Pinus roxburghii Sargent & Resin & Healing foot cracks \\
\hline Podophyllum hexandrum Royle. & Root & Antiseptic, wounds \\
\hline Polygonatum verticillatum (L.) All. & Root & Gastric problems \\
\hline Primula denticulata Sm. & Root and flower & Lice killing \\
\hline Prinsepia utilis Royle & Seed and roots & Stomach problems \\
\hline Pyrus pashia Buch.-Ham. ex D. Don & Fruits & Digestive disorders \\
\hline Ranunculus hirtellus Royle. & Whole plant & Wounds and cuts \\
\hline Reinwardtia indica Dumort. & Flower juice & Mouth wash \\
\hline Rhododendron arboreum Smith & Flower juice & Health tonic \\
\hline Roscoea alpina Royle & Root & Urinary infections \\
\hline Rubia manjith Roxb. ex Fleming & Flowers & Health tonic \\
\hline Rubus nepalensis (Hook.f.) Kuntze & Root & Burns \\
\hline Sapindus mukorossi Gaertn. & Fruit and seeds & Hair and antiseptic \\
\hline Sarcococca saligna (D.Don) Muell.-Arg. & Leaves & Bone and muscle pains \\
\hline Silene edgeworthii Bocquet. & Tender plant parts & Eye infections \\
\hline Swertia chirayita (Roxb. ex Fleming) Karsten & Leaves & Fever and blood diseases \\
\hline Synotis alatus (Wall. ex DC.) C. Jeffrey \& Chen. & Whole plant & Fever \\
\hline Taxus baccata $\mathrm{L}$. & Bark & Breast infection \\
\hline Urena lobata L. & Root & Muscle pains \\
\hline Urtica ardens Link. & Leaf and seeds & Skin and hair diseases \\
\hline
\end{tabular}


Table $\mathbf{5}$ Medicinal uses of plant species reported from the present study area (Continued)

\begin{tabular}{lll}
\hline Urtica dioica $\mathrm{L}$. & leaves & Hair wash \\
\hline Veronica anagallis-aquatica Linn. & Whole plant & Wounds and burns \\
\hline Zanthoxylum armatum DC & Bark & Tooth ache \\
\hline
\end{tabular}

the Panwalikantha at an elevation of $3800 \mathrm{~m}$. [45] reported a total of 335 medicinal plant species from the high altitude cold desert areas of Lahul-Spiti in Ladkh of which 45 were rare and endangered. [46] reported 228 species with medicinal and aromatic properties from Renuka Wildlife Sanctuary of Himalaya. Many of these medicinal plants are under of threat due to their heavy extraction [47]. A total of 1748 species having medicinal value have been reported from Indian Himalayan Region [15] contributing $90 \%$ of raw material for herbal industries in India and for export [48]. World trade figures suggest that India ranks next to China exporting raw material of medicinal plants [49].

The most commonly used parts of ethnomedicinal plants as collected through different literature survey were leaves $(32 \%)$, roots $(24 \%)$, whole plants or plant (13\%), followed by fruits (9\%) and seeds and flowers $(6 \%$ each). This corroborates with [50] who also found that leaves were the most frequently used plant parts (48\%) followed by stem bark (16\%), roots and root bark (10\%), while the fruits, whole plant, and aerial parts accounted for less than $10 \%$ for each. [19] reported that a single plant may be used for curing more than one ailment and observed that roots and root based preparations are the most used plant parts. [51] reported from Nepal that bark, flower, fruit, leaf, root, rhizome, tuber, seed, shoot, resin, and wood were used in this sequence.

In the study eighteen endangered plant species were found. [52] reported 37 species from Nanda Devi Biosphere Reserve as critically endangered, endangered, vulnerable and low risk near threatened using IUCN criteria. [15] reported that, as a result of over extraction $3.5 \%$ of the medicinal plants of the Indian Himalayan Regions (IHR) fall in different categories of threats.

In the study, the density and frequency for Aconitium heterophyllum was 0.33 plants $/ \mathrm{m}^{2}$ and $18.75 \%$ while for Jurinea dolomiaea it was 0.08 plants $/ \mathrm{m}^{2}$ and $3.75 \%$ respectively. Picrorhiza kurrooa was found with density of $0.23 \mathrm{ind} / \mathrm{m}^{2}$ having frequency of $12.50 \%$ while Podophyllum hexandrum with 0.19 plants $/ \mathrm{m}^{2}$ density and $11.25 \%$ frequency. [53] reported $0.33 \mathrm{ind} / \mathrm{m}^{2}$ density of Aconitium heterophyllum in a part of Kedarnath Wildlife Sanctuary and [54] in Gori valley reported a total $0.465 \mathrm{ind} / \mathrm{m}^{2}$, and [55] found a density of $1.0 \mathrm{ind} / \mathrm{m}^{2}$ at Hari Ki Dun area to 2.57 ind $/ \mathrm{m}^{2}$ in Tungnath area of Garhwal Himalaya. [56] reported 2.721 ind $/ \mathrm{m}^{2}$ and $86 \%$ values of density and frequency for Jurinea dolomiaea in alpine meadows of Kumaun Himalaya. [57] reported a density of $7 \mathrm{ind} / \mathrm{m}^{2}$ and frequency of $100 \%$ for Jurinea dolomiaea in rocky areas in alpine area of Chhota Bhangal in Himachal Pradesh. Working on the population density of Picrorhiza kurrooa, [54] reported a density of $3.89 \mathrm{ind} / \mathrm{m}^{2}$ from upper Gori valley and $4.5 \mathrm{ind} / \mathrm{m}^{2}$ in the valley of Flowers National Park, while [53] reported density values of 3.36 ind $/ \mathrm{m}^{2}$ in Kedarnath Wildlife Sanctuary. [47] reported 2 ind $/ \mathrm{m}^{2}$ density of Podophyllum hexandrum in Pin Valley National Park, while [58] reported 21.8 to 94.73 ind $/ \mathrm{m}^{2}$ density and [54] reported $0.193 \mathrm{ind} / \mathrm{m}^{2}$ in Gori valley, with a density of $0.98 \mathrm{ind} / \mathrm{m}^{2}$ in the Valley of Flowers National Park and 0.72 ind $/ \mathrm{m}^{2}$ in Kedarnath Wildlife Sanctuary. In contrast [59] found only density values of $0.012 \mathrm{ind} / \mathrm{m}^{2}$ and a frequency value of $18.70 \%$ in its natural habitats in Kashmir Himalaya.

The study indicates that in-depth phytochemical and pharmacological investigations would be of interest for some plants with unique or lesser known medicinal applications. The conservation of plant biodiversity in the Indian Himalayan region has become a major concern and more detailed studies on population structure and regeneration rates are needed to plan conservation measures. The traditional knowledge of plant species as medicine is vanishing rapidly, and traditional health care systems are disappearing, and the oral transmittion of knowledge is clearly decreasing. Therefore, the knowledge of indigenous uses of native plants needs to be studied before it gets extinct [60].

\section{Conclusions}

Considering the ecological importance and population status of important ethnomedicinal species, we recommend the preparation of micro-plans for each important medicinal species, including data on best harvesting practice and quantity to be harvested. Most of this data is unknown for most medicinal plants. Propagation of plants using tissue culture techniques and conventional methods to allow for their transplantation into natural habitats and niche areas of the species will be an important step towards their conservation. Additional ecological studies, including population assessments using standard ecological methods are needed to effectively plan the conservation and management for threatened, rare and endangered species. The development of agroproduction techniques for certain species of Garhwal Himalaya can help to meet the requirement of raw material for commercial use and reduce the pressure on the existing populations in natural habitats. 


\section{Competing interests}

The authors declare that they have no competing interests.

\section{Authors' contributions}

JAB and MK complied the collected field data, analysed and draft the manuscript, RWB revised the manuscript added the valuable suggestions for manuscript improvement. All authors read and approved the final manuscript.

\section{Acknowledgments}

The authors are thankful to the villagers of the study area to provide valuable knowledge about ethnomedicinal plants. Authors are also thankful to Dr Prashant Pusalkar (BSI) and Dr R. M. Panuli (GUH) for their help in identification of the plants specimen.

\section{Author details}

${ }^{1}$ Department of Forestry and Natural Resources, H.N.B, Garhwal University, Srinagar-Garhwal, Uttarakhand 249161, India. William L. Brown Center, Missouri Botanical Garden, P.O. Box 299, St. Louis, MO 63166-0299, USA.

Received: 18 July 2012 Accepted: 28 December 2012

Published: 2 January 2013

\section{References}

1. Shiva MP: Inventory of forestry resources for sustainable management and biodiversity conservation. New Delhi: Indus Publishing Company; 1996.

2. Prajapati ND, Purohit SS, Sharma AK, Kumar T: A handbook of medicinal plants. Jodhpur: Agrobios; 2003.

3. Kala CP: Current status of medicinal plants used by traditional Vaidyas in Uttaranchal state of India. Ethnobot Res Appl 2005, 3:267-278.

4. Kala CP: Medicinal plants of Indian trans-Himalaya. Dehradun: Bishen Singh Mahendra Pal Singh; 2002.

5. Farooquee NA, Majila BS, Kala CP: Indigenous knowledge systems and sustainable management of natural resources in a high altitude society in Kumaun Himalaya, India. J Hum Ecol 2004, 16:33-42.

6. Heywood VH: Global biodiversity assessment. Cambridge, UK: Cambridge University Press; 1995:75.

7. Loreau M, Oteng-Yeboah A: Diversity without representation. Nature 2006, 422:245.

8. Naranjo P: Urgent need for the study of medicinal plants. In Ethnobotany: evolution of a discipline. Portland: Dioscorides Press; 1995:392.

9. Mukherjee PK: Exploring green resources for drug development through ethnobotany. In Chemistry for green environment. New Delhi: Narosa Publishing House; 2005:98.

10. Utkarsh G: Patenting life? biodiversity and intellectual property rights. Resonance 2001, 2:51.

11. Shinwari SK, Gilani SS: Sustainable harvest of medicinal plants at Bulashbar Nullah, Astore (Pakistan). J Ethnopharmacol 2003, 84:289.

12. Rao MR, Palada MC, Becker BN: Medicinal and aromatic plants in agroforestry systems. Agrofor Syst 2004, 61:107-122.

13. Azaizeh HS, Fulder K, Khalil SO: Ethnomedicinal knowledge of local Arab practitioners in the Middle East Region. Fitoterapia 2003, 74:98-108.

14. Singh DK, Hajra PK: Floristic diversity. In Changing perspective of biodiversity status in the Himalaya. Edited by Gujral GS, Sharma V. New Delhi: British Council Division, British High Commission Publ. Wildlife Youth Services; 1996:23-38.

15. Samant SS, Dhar U, Palni LMS: Medicinal plants of Indian Himalaya: diversity distribution potential values. Almora: G.B. Pant Institute of Himalayan Environment and Development; 1998.

16. Singh D, Srivastava RK, Khanduri VP: Marketing strategies and trade of medicinal plants in Uttaranchal: Present and future prospects. Indian Forester 2005, 131(3):330-340.

17. Kala CP: Assessment of species rarity. Curr Sci 2004, 86(8):1058-1059.

18. Kala CP, Mathur VB: Patterns of plant species distribution in the transHimalayan region of Ladakh, India. J Vegetation Sci 2002, 13:751-754.

19. Singh G, Rawat GS: Ethnomedicinal survey of Kedarnath wildlife sanctuary in western Himalaya, India. Ind J Fundam Appl Life Sci 2011, 1:35-36.

20. Dhar U, Rawal RS, Upreti J: Setting priorities for conservation of medicinal plants- a case study in the Indian Himalaya. Biol Conserv 2000, 95:57-65.

21. Singh MP, Dey S: Indian medicinal plants. India: Satish Serial Publishing House, Delhi; 2005
22. Berkes F, Colding J, Folke C: Rediscover of traditional ecological knowledge as adaptive management. Ecol App/ 2000, 10:1251-1262.

23. Huntingto HP: Using traditional ecological knowledge in science: methods and application. Ecol App/ 2000, 10:1270-1274.

24. Cunningham AB: Applied ethnobotany. People, wild plant Use and conservation. London: Earthscan; 2001:300.

25. Muller-Dombios DJ, Ellenberg H: Aims and methods in vegetation ecology. New York: John Wiley and Sons; 1974

26. Mishra R: Ecology work book. Calcutta: Oxford and IBM publishing Co; 1968:244.

27. Gaur RD: Flora of the district Garhwal, North West Himalaya with Ethnobotanical Notes. India: Transmedia publications Srinagar (Garhwal) - U. P; 1999.

28. Joshi M, Kumar M, Bussmann RW: Ethnomedicinal uses of plant resources of the HaigadWatershed in kumaun Himalaya, India. Med Aromatic Plant Sci Biotechnol 2010, 4(Special issue):43-46.

29. IUCN: Draft IUCN Red. List categories. Gland, Switzerland: IUCN; 1993.

30. NMPB (National Medicinal Plant Board): Conservation assessment and management prioritisation of selected medicinal plants in western Himalayas. Himachal Pradesh, India: Shimlam: Workshop; 2003:22-26.

31. Semwal DP, Saradhi PP, Nautiyal BP, Bhatt AB: Current status, distribution and conservation of rare and endangered medicinal plants of Kedarnath Wildlife Sanctuary. Central Himalayas. Ind Curr Sci 2007, 92(12):1733-1738.

32. Naithani BD: Flora of chamoli. Vols.I-II. Calcutta: Botanical Survey of India; 1984-1985.

33. Murthy KRK: A pictorial field guide- floral gallery of Himalayan valley of flowers and adjacent areas. Chennai: Sudarshan Graphics Pvt. Ltd.; 2011.

34. Jain SK, Rao RR: A handbook of field and herbarium methods. New Delhi: Today and Tommorow; 1977

35. Uniyal BP, Sharma JR, Choudhary U, Singh DK: Flowering plants of uttarakhand (a checklist). Dehradun, India: Bishen Singh Mahendra Pal Singh; 2007.

36. Khumbongmayum AD, Khan ML, Tripathi RS: Survival and growth of seedlings of a few tree species in the four sacred groves of Manipur, Northeast India. Curr Sci 2005, 88(11):1781-1788.

37. CITES: (Convention on International Trade in Endangered Species Of Wild Flora and Fauna): The CITES appendices I, II and III; 2003. Available at: http://www.cites.org/eng/append/index.shtml.

38. Dhar U, Manjkhola S, Joshi M, Bhatt A, Bisht AK, Joshi M: Current status and future strategy for development of medicinal plants sector in Uttaranchal, India. Curr Sci 2002, 83(8):956-964.

39. Samant SS, Jitendra SB, Sharma A: Assessment of diversity, distribution, conservation status and preparation of management plan for medicinal plants in the catchment area of Prabati Hydroelectric Project Stage-III in Northwestern Himalaya. J Mt Sci 2007, 4(1):34-56.

40. Dhyani PP, Kala CP: Current research on medicinal plants: five lesser unknown but valuable aspects. Curr Sci 2005, 88(3):335

41. Joshi HC, Arya SC, Samant SS: Diversity, distribution and indigenous uses of plant species in Pindari area of Nanda Devi Biosphere Reserve- II. Ind J Forestry 2000, 24(4):514-536.

42. Samant SS, Pal S: Diversity and conservation status of medicinal plants in Uttaranchal State. Indian Forester. 2003, 129(9):1090-1108.

43. Mamgain SK, Rao RR: Some medicinal plants of Pauri Garhwal Himalaya. J Econ Taxon Bot 1990, 14(3):623-640.

44. Nautiyal BP, Pandey N, Bhatt AB: Analysis of vegetation pattern in an alpine zone in Northwest Himalayas: a case study of Garhwal Himalaya with reference to diversity and distribution patterns. Int J Ecol Environ Sci 1997, 23(1):49-65.

45. Kala CP: Medicinal plants of the high altitude cold desert in India: diversity, distribution and traditional uses. Int J Biodivers Sci Manag 2006, 2(1):43-56.

46. Subramani SP, Jishtu V, Verma RK, Kapoor KS: Floristic composition, lifeforms and biological spectrum of Renuka Wildlife Sanctuary, Himachal Pradesh. Ind Forester 2007, 133(1):79-92.

47. Kala CP: Status and conservation of rare and endangered medicinal plants in the Indian Trans Himalaya. Biol Conserv 2000, 93:371-379.

48. Ved DK, Mudappa A, Shanker D: Regulating export of endangered medicinal plant species-need for scientific vigour. Curr Sci 1998, 75:341-344.

49. Lange D: Trade figures for botanical drugs world-wide. Med Plant Conserv News/ 1997, 3:16-17.

50. Keter LK, Mutiso PC: Ethnobotanical studies of medicinal plants used by traditional health practitioners in the management of diabetes in lower eastern province, Kenya. J Ethnopharmacol 2012, 139:74-80. 
51. Kunwar RM, Nepal BK, Kshhetri HB, Rai SK, Bussmann RW: Ethnomedicine in Himalaya: a case study from Dolpa, Humla, Jumla and Mustang districts of Nepal. J Ethnobiol Ethnomed 2006, 2:27.

52. Samant SS, Joshi HC: Plant diversity and conservation status of Nanda Devi National Park and comparison with highland Natioanl Parks of the Indian Himalayan Region. Int J Biodivers Sci Manag 2005, 1:65-73.

53. Kala CP: Indigenous uses, Population density, and conservation of threatened medicinal plants in protected areas of Indian Himalaya. Conserv Biol 2005, 19(2):368-378.

54. Uniyal SK, Awasthi A, Rawat GS: Current Status and distribution of commercially exploited medicinal and aromatic plants in upper Gori valley, Kumaun Himalaya, Uttaranchal. Curr Sci 2002, 82(10):1246-1252.

55. Nautiyal S, Rao KS, Maikhuri RK, Negi KS, Kala CP: Status of medicinal plants on way to Vashuki Tal in Mandakini Valley, Garhwal, Uttaranchal. J Non-Timber Forest Prod 2002, 9:124-131.

56. Awasthi A, Uniyal SK, Rawat GS: Status and extraction patterns of Jurinea dolomiaea Boiss. (dhoop) in an alpine meadow of Kumaon Himalaya (Uttaranchal). Indian Forester 2003, 129(5):589-595.

57. Uniyal SK, Singh KN, Jamwal P: Lal Brij: Traditional use of medicinal plants among the tribal communities of Chhota Bhangal, Western Himalaya. J Ethnobiol Ethnomed 2006, 2:14.

58. Airi S, Rawal RS, Dhar U, Purohit AN: Population studies on Podophyllum hexandrum Royle- a dwindling, medicinal plant of the Himalaya. Plant Genet Resour Newsl 1997, 110:29-34.

59. Haleema A, Gupta RC, Siddique MAA, Bhat MA, Nazki IT: Distribution, frequency, abundance and density of Podophyllum hexandrum Royle in Anantanag and Srinagar Districts of Kashmir Himalayas. J Plant Sci Res 2006, 22:33-35.

60. Kaul MK, Singh V, Sharma PK, Bhatia AK: Ethnobatonic studies in NorthWest and Trans-Himalaya II. Approaches to study of ethno botany towards the human welfare in remote North-West and Trans-Himalayas. J Econ Taxonomic Bot 1990, 14(2):271-285.

doi:10.1186/1746-4269-9-1

Cite this article as: Bhat et al:: Ecological status and traditional knowledge of medicinal plants in Kedarnath Wildlife Sanctuary of Garhwal Himalaya, India. Journal of Ethnobiology and Ethnomedicine 2013 9:1.

\section{Submit your next manuscript to BioMed Central and take full advantage of:}

- Convenient online submission

- Thorough peer review

- No space constraints or color figure charges

- Immediate publication on acceptance

- Inclusion in PubMed, CAS, Scopus and Google Scholar

- Research which is freely available for redistribution 